\title{
Corpus-based Studies on Nursing Textbooks
}

\author{
Alif Fairus Nor Mohamad \\ Universiti Putra Malaysia \\ $\mathrm{Ng}$ Yu Jin (corresponding author) \\ Universiti Tenaga Nasional, Malaysia \\ E-mail: ngyujin6@gmail.com
}

Doi:10.7575/aiac.alls.v.4n.2p.21
URL: http://dx.doi.org/10.7575/aiac.alls.v.4n.2p.21

Received: 05/04/2013

Accepted: 04/06/2013

\begin{abstract}
English for Specific Purposes (ESP) educators often face dilemma in deciding what lexical items to teach their students. In the field of English for Nursing Purposes (ENP), there is no exception on this issue as well. Only by analyzing the nursing corpus made up of essential core textbooks that can provide better insights and guide to both nursing students and educators. This research aims to highlight the 2,000 most frequently used nursing words across the core textbooks of nursing and to profile the types of 'low frequency' lexis which comprise the nursing corpus in terms of the General Service List (GSL) and Academic Word List (AWL) lexis coverage. By knowing the frequently used nursing words would further reduce students' reading deficiency if the students use the 2000-word list.
\end{abstract}

Keywords: Corpus-based studies on nursing textbooks; Most frequently used nursing content words; Low-frequency words profiling using the GSL and AWL; Nursing lexis

\section{Introduction}

The primary function of a nursing corpus should directly assist nurses in understanding the language for the purpose of communication with each other and their patients, report writing and interpreting information for work purposes. It is important to note here that the identification of the most frequent field-specific academic words is essential (Chung \& Nation, 2003); for responsible planning for ESP or EAP courses. The field of English for Academic Purposes (EAP) such as ENP has become imperative in bridging the students from their general proficiency English into learning the language in a context. This scenario has become crucial especially when students enroll to higher learning institutions in Malaysia. Most of the nursing colleges in this country have developed an intensive program with the hope to solve this matter.

Unfortunately, the English instructors who are responsible in teaching the students may also encounter difficulties; not knowing the vocabulary to be focused on as they are not from the nursing background. Having a good knowledge of academic vocabulary has been emphasized for success at higher levels of education (Ward, 2009; Nation, 2001; Coxhead, 2000; Nation \& Waring, 1997). Thus, the best solution to overcome this matter would be by looking at a corpus of the field in order to 'undergo and understand' the way the vocabulary is presented in the nursing text. On the other hand, the field of nursing is exclusive in the sense that they have a specialized set of vocabulary which is distinct from other fields. The analysis of the academic English corpora is able to portray the vocabulary types, frequency and range coverage of the texts in different fields which lead to the production of word lists (Nation, 2001).

This study focuses on the vocabulary used in a nursing text of 3,640,760 running words from 7 major academic books used by student nurses for a 3 years diploma programme in Malaysia. The academic course books are chosen based on the syllabus provided by the Nursing Board of Malaysia. It is essential to observe the different types of vocabulary used in these course books as it would assist the students to be able to comprehend a nursing text in a more effective way.

1.1 Rationale behind the Development of the Corpus

There is reasonable consensus that a corpus will not just provide insights into the contents but also that the results of the analyses will be claimed to be typical of the language from which the corpus was selected (Mukundan \& Menon 2007). L2 students who need to use the language for challenging academic purposes, such as reading academic texts or reading technical texts and who normally have only a limited amount of time to do this, need systematic vocabulary learning instruction in addition to learning through extensive reading or incidental learning (Coady,1997, Schmitt, 2000 \& Nation, 2001).

The creation of nursing corpus in this study is essential as it will reveal the specialized vocabulary that must be instilled in the students. The need for specific academic corpora for various fields is more eminent than ever now as the availability of language corpora to learners and teachers facilitates better learning (Mudraya, 2006).

The corpus created will not only help student nurses but also facilitates the lecturers and instructors in the process of teaching and learning. According to Holme (2006), a common complaint among English language teachers faced with unfamiliar scientific content is that they do not fully understand what they are dealing with and are not properly 
equipped to explain the meanings that confront them. With the existence of the corpus, lecturers and instructors would be able to prepare themselves with the proper subject matter.

\subsection{Books Used in Creating the Corpus}

This corpus is created based on seven course books as listed below:

i) Anatomy \& Physiology

ii) Medical Surgical Nursing

iii) Pharmacology in Nursing

iv) Clinical Nursing and Related Sciences

v) Fundamental of Nursing

vi) Nursing Ethics

vii) Leadership and Management in Nursing

The course books listed are categorized into 2 which are:

a) Specialized Technical Nursing Course Books (i, ii, iii, iv and v)

b) Non Technical Nursing Course Books (vi and vii)

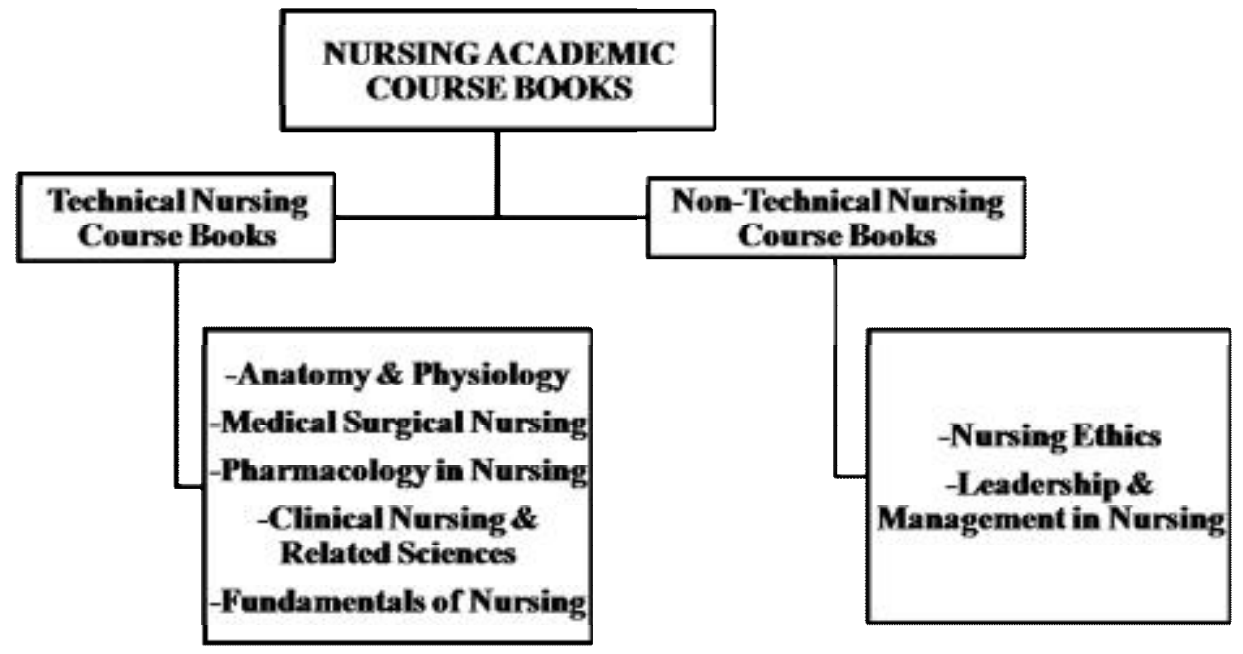

Figure 1. The Categorization of the Books

The first book is named "Ross and Wilson Anatomy and Physiology in Health and Illness" by Anne Waugh and Allison Grant. This subject will be able to help learners understand the basic human body functions and life processes, major human body systems and their functions and also the anatomical locations, structures, and physiological functions of the main components of each major system of the human body. The second book is named "Brunner \& Suddarth's Textbook of Medical-Surgical Nursing" written by Suzanne C. Smeltzer, Brenda G. Bare, Janice L. Hinkle and Kerry $\mathrm{H}$. Cheever. In this course, student nurses will receive advance education not only in medical but in surgical as well in order to expand the nursing roles in hospitals and healthcare centres. The third book is "Trounce's Clinical Pharmacology for Nurses" by Ben Greenstein. Pharmacology is the study of the science of medicine. In the healthcare field, these drugs are used to treat, prevent or even diagnose diseases and therefore insufficient knowledge on this by doctors or nurses might lead to a worsen condition or even fatality. The fourth book is "Watson's Clinical Nursing and Related Sciences", $7^{\text {th }}$ edition, edited by Mike Walsh and Alison Crumbie.

The clinical nursing skills for the nurses are essential not only to provide comprehensive care but also enhance clinical competence. For nurses to provide health service at different settings such as hospitals, health centers, health post and at the community level including home based care for chronically sick patients, this course is very necessary. The fifth book was named "Kozier and Erb's Fundamentals of Nursing, Concepts, Process and Practice," $8^{\text {th }}$ Edition written by Berman, Snyder, Kozier and Erb. This subject is a general idea of patient relations including basic human needs, communication skills, stress and hospitalization, and transcultural factors influencing nursing care; the adult patient care unit; advanced principles of patient hygiene; body mechanics; positioning and ambulating the adult patient; and medical 
isolation. The sixth is the "Nursing Ethics" $5^{\text {th }}$ Edition written by Ian E. Thompson, Kath M. Melia, Kenneth M Boyd, and Dorothy Horsburgh.

Nursing ethics is a branch of applied ethics that concerns itself with activities in the area of nursing. Although nursing ethics are quite similar to medical ethics, it can be differentiated by the highlights on relationship, human dignity and mutual care. It is important to study nursing ethics specifically because student nurses will occupy a specific place in the division of labour in healthcare. The seventh book chosen was "Effective Leadership and Management in Nursing" by Eleanor J.Sullivan and Phillip J. Decker. This course provides full details on the leadership and management in nursing profession. Leadership is one of the skills that student nurses should possess which will help them provide direct care to the community.

\section{Objectives of the Study}

The main aim of this study is to explore and determine the language of nursing from the aspects of the vocabulary used in terms of their loading, distributions and the specialized words in the nursing texts. Thus, the objectives of the study are as below:

1) To identify the word properties in the language of nursing

2) To determine the 2000 most frequently used nursing words

\section{Methodology}

The seven academic nursing texts were used to create the nursing corpus which contains 3,640,760 running words. These seven books were chosen based on the program of study for the nursing diploma course in one of the nursing college in Malaysia. These textbooks were then scanned, digitized and all the charts, diagrams and bibliographies were removed and convert all into text file format (.txt). For this step, Abby Fine Reader 8.0 software was used to preserve the originality of the texts. The scanning and digitizing procedure has been done in various studies by AlMarooqiMukundan (2009), Mukundan (2007), and Mukundan \& Hussin (2007) and Mukundan and Menon (2007). The digitized texts were run using RANGE software (Heatley, Nation \& Coxhead, 2002) to discriminate the types of words according to word list (GSL, AWL and non-word list items). Subsequently, Word Smith 5.0 (Scott, 2010) was used to find the 2,000 most frequently used nursing words and the number of low frequency words in each nursing academic books.

\section{Results and Discussion}

\subsection{The Vocabulary Size of the Corpora}

A total of 3,640,760 tokens (running words) were found from all the texts inclusive of the specialized (technical) and general (non-technical) nursing academic course books. The corpus presents 97,836 types of words. The tokens and types represented in each book are shown in the table below.

Table 1. The number of tokens and types in each book.

\begin{tabular}{llrr}
\hline \multicolumn{1}{c}{ CORPUS } & TOKENS & \multicolumn{1}{c}{ TYPES } \\
\hline i) & Anatomy \& Physiology & 195,729 & 11,182 \\
ii) & Medical Surgical Nursing & $1,529,951$ & 64,592 \\
iii) & Pharmacology in Nursing & 219,366 & 19,101 \\
iv) & Clinical Nursing and Related Sciences & 664,979 & 25,710 \\
v) & Fundamental of Nursing & 623,097 & 30,681 \\
vi) & Nursing Ethics & 241,773 & 12,065 \\
vii) Leadership and Management in Nursing & 165,865 & 11,420 \\
& $\quad$ TOTAL & $3,640,760$ &
\end{tabular}

In this study, the low frequency words listed below are defined as the words which occur once up to ten times in each book. The words are then identified and categorized into three:

1. Words which are in General Service List (GSL)

2. Words which are in the Academic Word List (AWL)

3. Words which are not in the General Service List (GSL) and Academic Word List (AWL)

The motive to identify the number of these words and their categories is to show the coverage of words in the nursing academic text. 


\begin{tabular}{ccccc}
\hline FREQUENCY & TOTAL WORDS & \multicolumn{3}{c}{ LIST } \\
\cline { 3 - 4 } & & GSL & AWL & NON \\
& & & & GSL/AWL \\
$\mathbf{1}$ & 4401 & 594 & 254 & 3553 \\
$\mathbf{2}$ & 1509 & 326 & 136 & 1047 \\
$\mathbf{3}$ & 863 & 199 & 89 & 575 \\
$\mathbf{4}$ & 580 & 129 & 52 & 399 \\
$\mathbf{5}$ & 443 & 108 & 56 & 279 \\
$\mathbf{6}$ & 309 & 87 & 34 & 188 \\
$\mathbf{7}$ & 290 & 85 & 29 & 176 \\
$\mathbf{8}$ & 209 & 70 & 33 & 106 \\
$\mathbf{9}$ & 189 & 53 & 18 & 118 \\
$\mathbf{1 0}$ & 144 & 44 & 19 & 81 \\
\hline
\end{tabular}

Table 3. Book of Medical-Surgical Nursing

\begin{tabular}{ccccc}
\hline FREQUENCY & TOTAL WORDS & \multicolumn{3}{c}{ LIST } \\
\cline { 3 - 4 } & & GSL & AWL & NON \\
GSL/AWL & & & 36505 \\
$\mathbf{1}$ & 37439 & 699 & 235 & 6296 \\
$\mathbf{3}$ & 6885 & 427 & 162 & 2930 \\
$\mathbf{4}$ & 3335 & 290 & 115 & 1943 \\
$\mathbf{5}$ & 2263 & 244 & 76 & 1317 \\
$\mathbf{6}$ & 1575 & 164 & 94 & 959 \\
$\mathbf{7}$ & 1180 & 155 & 66 & 738 \\
$\mathbf{8}$ & 945 & 139 & 68 & 581 \\
$\mathbf{9}$ & 772 & 136 & 55 & 500 \\
$\mathbf{1 0}$ & 671 & 118 & 53 & 395 \\
\hline
\end{tabular}

Table 4. Book of Pharmacology in Nursing

\begin{tabular}{ccccc}
\hline FREQUENCY & TOTAL WORDS & \multicolumn{3}{c}{ LIST } \\
\cline { 3 - 4 } & & GSL & AWL & NON \\
$\mathbf{1}$ & 10470 & 826 & 344 & 9300 \\
$\mathbf{2}$ & 2376 & 390 & 166 & 1820 \\
$\mathbf{3}$ & 1254 & 261 & 106 & 887 \\
$\mathbf{4}$ & 819 & 208 & 83 & 528 \\
$\mathbf{5}$ & 535 & 144 & 57 & 334 \\
$\mathbf{6}$ & 394 & 109 & 37 & 248 \\
$\mathbf{7}$ & 354 & 109 & 31 & 214 \\
$\mathbf{8}$ & 246 & 74 & 36 & 136 \\
$\mathbf{9}$ & 221 & 78 & 25 & 118 \\
$\mathbf{1 0}$ & 186 & 61 & 23 & 102 \\
\hline
\end{tabular}




\begin{tabular}{ccccc}
\hline FREQUENCY & TOTAL WORDS & \multicolumn{3}{c}{ LIST } \\
\cline { 3 - 4 } & & GSL & AWL & NON \\
$\mathbf{1}$ & 10661 & & & GSL/AWL \\
$\mathbf{2}$ & 3409 & 765 & 335 & 9561 \\
$\mathbf{3}$ & 1864 & 481 & 215 & 2713 \\
$\mathbf{4}$ & 1282 & 340 & 151 & 1373 \\
$\mathbf{5}$ & 870 & 255 & 112 & 915 \\
$\mathbf{6}$ & 735 & 176 & 99 & 595 \\
$\mathbf{7}$ & 561 & 163 & 86 & 486 \\
$\mathbf{8}$ & 467 & 134 & 63 & 364 \\
$\mathbf{9}$ & 376 & 108 & 60 & 299 \\
$\mathbf{1 0}$ & 310 & 102 & 45 & 229 \\
\hline
\end{tabular}

Table 6. Book of Fundamentals of Nursing

\begin{tabular}{ccccc}
\hline FREQUENCY & TOTAL WORDS & \multicolumn{3}{c}{ LIST } \\
\cline { 3 - 4 } $\mathbf{1}$ & & GSL & AWL & NON \\
& & & & GSL/AWL \\
$\mathbf{2}$ & 14619 & 828 & 303 & 13488 \\
$\mathbf{3}$ & 3762 & 450 & 210 & 3102 \\
$\mathbf{4}$ & 2101 & 334 & 141 & 1626 \\
$\mathbf{5}$ & 1399 & 265 & 118 & 1016 \\
$\mathbf{6}$ & 981 & 212 & 92 & 677 \\
$\mathbf{7}$ & 772 & 179 & 76 & 517 \\
$\mathbf{8}$ & 608 & 143 & 66 & 399 \\
$\mathbf{9}$ & 525 & 138 & 62 & 325 \\
$\mathbf{1 0}$ & 417 & 113 & 54 & 250 \\
& 367 & 101 & 57 & 209 \\
\hline
\end{tabular}

Table 7. Book of Nursing Ethics

\begin{tabular}{ccccc}
\hline FREQUENCY & TOTAL WORDS & \multicolumn{3}{c}{ LIST } \\
\cline { 3 - 5 } & & GSL & AWL & NON \\
& & & & GSL/AWL \\
$\mathbf{1}$ & 5078 & 787 & 380 & 3911 \\
$\mathbf{2}$ & 1694 & 452 & 216 & 1026 \\
$\mathbf{3}$ & 987 & 290 & 170 & 527 \\
$\mathbf{4}$ & 574 & 195 & 98 & 281 \\
$\mathbf{5}$ & 453 & 157 & 85 & 211 \\
$\mathbf{6}$ & 326 & 134 & 70 & 122 \\
$\mathbf{7}$ & 243 & 117 & 43 & 83 \\
$\mathbf{8}$ & 218 & 87 & 56 & 75 \\
$\mathbf{9}$ & 161 & 79 & 42 & 40 \\
$\mathbf{1 0}$ & 146 & 71 & 37 & 38 \\
\hline
\end{tabular}




\begin{tabular}{|c|c|c|c|c|}
\hline \multirow[t]{2}{*}{ FREQUENCY } & \multirow[t]{2}{*}{ TOTAL WORDS } & \multicolumn{3}{|c|}{ LIST } \\
\hline & & GSL & AWL & $\begin{array}{c}\text { NON } \\
\text { GSL/AWL }\end{array}$ \\
\hline 1 & 4851 & 802 & 385 & 3664 \\
\hline 2 & 1604 & 418 & 197 & 989 \\
\hline 3 & 890 & 274 & 139 & 477 \\
\hline 4 & 626 & 205 & 134 & 287 \\
\hline 5 & 413 & 156 & 75 & 182 \\
\hline 6 & 323 & 110 & 68 & 145 \\
\hline 7 & 276 & 116 & 59 & 101 \\
\hline 8 & 201 & 101 & 45 & 55 \\
\hline 9 & 181 & 78 & 30 & 73 \\
\hline 10 & 160 & 70 & 38 & 52 \\
\hline
\end{tabular}

Tables 2 to 8 show the number of words which occur once up to 10 times in all the nursing academic course books. From the data presented, it is revealed that there are many words in the nursing academic text which do not belong to the general or academic words. These words are the specialized vocabulary in the nursing field and they may consist of medical terms used in the environment or work place of the nurses. This further enhances the rationale of having a corpus for the language of nursing as it is able to expose underlying words in the nursing field. These 'low-frequency' words may bring about lexical learning challenges to student nurses due to their low occurrence in the textbooks. The GSL and AWL can be easily obtained or downloaded if they are required by the educators and students. However, these non-GSL and non-AWL words cannot be identified unless further content analysis or lexicology research is carried out. This result shows that even the specialised nursing words are not frequently recycled and repeated throughout the texts which may debilitate the effective learning of nursing lexis. Specialised nursing words should appear more frequently throughout the texts so that they can be reckoned with and learned more effectively. Perhaps, these textbooks have not accommodated the necessary lexical learning principles.

\subsection{The 2,000 most frequent nursing words}

The most common 2,000 words used in the nursing academic course books are identified after removing all the functional words in the English language such as articles, pronouns and other functional grammatical items in the language. The GSL word families were not removed at this stage because this 2000 words list is considered as a list to enhance the teaching and learning process. GSL words which are maintained in these 2,000 most frequent nursing words are the words which are considered as the words which are useful and needed for the field of ENP. Moreover, there are 2,157 types in these most frequent nursing words which eventually make it a higher coverage list compared to both GSL and AWL merged. Thus, it shows that a genre-specific corpus should always be developed in order to discriminate and identify the eminent words in the field that provide higher significance to content-specific word list development (Martinez,2009;Mudraya 2006;Ward,1999). The words found are remained in their lexico-grammar forms to promote better comprehension of words in 'authentic' or 'real' forms in terms of frequency (see Ward, 2009).

Table 9: 25 most frequent used nursing words in the nursing academic course books

\begin{tabular}{cc}
\hline WORDS & FREQUENCY \\
\hline PATIENT & 16982 \\
PATIENTS & 13934 \\
CARE & 11909 \\
HEALTH & 9177 \\
BLOOD & 8406 \\
NURSE & 8220 \\
NURSING & 7535 \\
PAIN & 7089 \\
USED & 6402
\end{tabular}


USE

DISEASE

5483

NURSES

TREATMENT

BODY

PEOPLE

CLIENT

INCLUDE

SKIN

PRESSURE

FAMILY

MANAGEMENT

TIME

RISK

INFECTION

CLIENTS
4979

4908

4646

4567

4412

4380

4156

4098

4081

3806

3783

3783

3721

3576

3455

Functional words like 'the', 'an' and ' $a$ ' (articles), 'she', 'he', 'them', etc (pronouns) and other functional grammatical items were removed from the list. The top 100 of the 2,000 nursing words is listed in Appendix A.

\section{Conclusion}

The teaching and learning of English for Nursing Purposes (ENP) can be enhanced with the existence of the nursing corpus. Student nurses may be exposed to the vocabulary important in their field in a more comprehensive way. With the creation of this corpus, ENP material developers would have an idea and guidelines on the vocabulary needed to be taken into consideration when developing a material or an ENP course book. On the other hand, the production of the nursing dictionaries can be developed not only based on the medical terminologies but also focusing on the frequently used nursing words in the field.

\section{References}

Chung, T.M., \& Nation, P. (2003). Technical vocabulary in specialized texts. Reading in a Foreign Language, 15(2), 103-116.

Coady, J. (1997). L2 Vocabulary Acquisition: A synthesis of the research. In J.Coady \& T. Huckin. Second

Language Vocabulary Acquisition (pp. 225 - 237). Cambridge: Cambridge University Press.

Coxhead, A. (2000). A New Academic Word List. TESOL Quarterly, 34(2):213-238.

Heatley, A., Nation, I.S.P. and Coxhead, A. 2002. RANGE and FREQUENCY programs. http://www.vuw.ac.nz/lals/staff/Paul_Nation

Holme, R. (2006). English for Science or Science for English: Designing content-based Materials for Language Teaching. In Reading on ELT Materials, ed. Mukundan, J. 17-34, Serdang: Universiti Putra Malaysia Press

Martinez, I.A., Beck S.C. \& Panza, C.B. (2009). Academic vocabulary in agriculture research articles: A corpus-based study. English for Specific Purposes, 28,183-198. Doi:10.1016/j.esp.2009.04.003

Mudraya, O. (2006). Engineering English: a lexical frequency instructional model. English for Specific Purposes, 25,235-256. Doi:10.1016/j.esp.2005.05.002

Mukundan, J. (2007). Irregularities in Vocabulary Load and Distribution in Same Level Textbooks Written By Different Writers. Indonesian JELT 3/1, 99- 118.

Mukundan, J.\& Menon, S. (2007). The Language of Mathematics, Science and English Language Textbooks: A Comparative Corpus Based Study. ELT Matters 4 (pp 244-271). Serdang: Universiti Putra Malaysia Press.

Mukundan, J. and Hussin, A (2007).A Forensic Study of Vocabulary Load and Distribution in Five Malaysian Secondary School English Textbooks (Forms 1-5). Pertanika J.Soc.Sci. \& Hum. 15(2): 59-74 
Mukundan, J. (2009). ESL Textbook Evaluation: A Composite Framework. LAP Lambert Academic Publishing.Mukundan, J. \& Menon, S. (2007). The Language of Mathematics, Science and English Language Textbooks: A Comparative Corpus Based Study. In ELT Matters 4 Developments in English Language Learning and Teaching, ed. Mukundan, J. \& Menon, S., pp. 244-271. Serdang: Universiti Putra Malaysia Press.

Nation, P. \& Waring, R. (1997). Vocabulary size, text coverage and word lists. In N.Schmitt \& M.McCarthy (Eds.). Vocabulary: Description, Acquisition and Pedagogy (pp. 6-19). Cambridge: Cambridge University Press.

Nation, P (2001). Learning Vocabulary in Another Language. Cambridge: Cambridge University Press.

Schmitt, N. (2000). Vocabulary in Language Teaching. Cambridge: Cambridge University Press.

Scott, M. (2010). Version 5.0 WordSmith Tools. Oxford: Oxford University Press.

Ward, J (1999). How a Large Vocabulary do EAP engineering students need? Reading in a Foreign Language, 12(2), 309-323.

Ward, J. (2009). A basic engineering English word list for less proficient foundation engineering undergraduates. English for Specific Purpose, 28, 170-182. 
Appendix A

\begin{tabular}{|c|c|}
\hline 1 & PATIENT \\
\hline 2 & PATIENTS \\
\hline 3 & CARE \\
\hline 4 & HEALTH \\
\hline 5 & BLOOD \\
\hline 6 & NURSE \\
\hline 7 & NURSING \\
\hline 8 & PAIN \\
\hline 9 & USED \\
\hline 10 & USE \\
\hline 11 & DISEASE \\
\hline 12 & NURSES \\
\hline 13 & TREATMENT \\
\hline 14 & BODY \\
\hline 15 & PEOPLE \\
\hline 16 & CLIENT \\
\hline 17 & INCLUDE \\
\hline 18 & SKIN \\
\hline 19 & PRESSURE \\
\hline 20 & FAMILY \\
\hline 21 & MANAGEMENT \\
\hline 22 & TIME \\
\hline 23 & RISK \\
\hline 24 & INFECTION \\
\hline 25 & CLIENTS \\
\hline 26 & SYSTEM \\
\hline 27 & SYMPTOMS \\
\hline 28 & NEED \\
\hline 29 & CAUSE \\
\hline 30 & CHANGES \\
\hline 31 & ASSESSMENT \\
\hline 32 & FLUID \\
\hline 33 & CELLS \\
\hline 34 & IMPORTANT \\
\hline 35 & FUNCTION \\
\hline 36 & NORMAL \\
\hline 37 & PERSON \\
\hline 38 & LOSS \\
\hline 39 & OCCUR \\
\hline 40 & INCREASED \\
\hline 41 & TISSUE \\
\hline
\end{tabular}

\begin{tabular}{|c|c|}
\hline 42 & SEE \\
\hline 43 & PROBLEMS \\
\hline 44 & THERAPY \\
\hline 45 & HEART \\
\hline 46 & SURGERY \\
\hline 47 & COMMON \\
\hline 48 & PROCESS \\
\hline 49 & LEVEL \\
\hline 50 & INFORMATION \\
\hline 51 & HOME \\
\hline 52 & RESULT \\
\hline 53 & PHYSICAL \\
\hline 54 & BOTH \\
\hline 55 & NEEDS \\
\hline 56 & CANCER \\
\hline 57 & SUPPORT \\
\hline 58 & PROVIDE \\
\hline 59 & SIGNS \\
\hline 60 & CLINICAL \\
\hline 61 & FACTORS \\
\hline 62 & EXAMPLE \\
\hline 63 & EFFECTS \\
\hline 64 & PREVENT \\
\hline 65 & ACUTE \\
\hline 66 & CONTROL \\
\hline 67 & SPECIFIC \\
\hline 68 & POSSIBLE \\
\hline 69 & MEDICATIONS \\
\hline 70 & CHRONIC \\
\hline 71 & WELL \\
\hline 72 & ASSOCIATED \\
\hline 73 & LEVELS \\
\hline 74 & OCCURS \\
\hline 75 & LIFE \\
\hline 76 & MUSCLE \\
\hline 77 & AGE \\
\hline 78 & MEDICAL \\
\hline 79 & BONE \\
\hline 80 & HELP \\
\hline 81 & YEARS \\
\hline 82 & RELATED \\
\hline
\end{tabular}

\begin{tabular}{|c|c|}
\hline 83 & RESPIRATORY \\
\hline 84 & PART \\
\hline 85 & PRACTICE \\
\hline 86 & CHANGE \\
\hline 87 & AREA \\
\hline 88 & FOLLOWING \\
\hline 89 & RATE \\
\hline 90 & WOMEN \\
\hline 91 & FIGURE \\
\hline 92 & WORK \\
\hline 93 & INCREASE \\
\hline 94 & LESS \\
\hline 95 & NECESSARY \\
\hline 96 & GIVEN \\
\hline 97 & FIRST \\
\hline 98 & SEVERE \\
\hline 99 & ACTIVITY \\
\hline 100 & USING \\
\hline 101 & RESPONSE \\
\hline 102 & OTHERS \\
\hline 103 & INJURY \\
\hline 104 & RIGHT \\
\hline 105 & DEVELOP \\
\hline 106 & POSITION \\
\hline 107 & HIGH \\
\hline 108 & URINE \\
\hline 109 & DRUGS \\
\hline 110 & HOURS \\
\hline 111 & APPROPRIATE \\
\hline 112 & RENAL \\
\hline 113 & RESULTS \\
\hline 114 & INCLUDING \\
\hline 115 & SMALL \\
\hline 116 & STATUS \\
\hline 117 & INDIVIDUAL \\
\hline 118 & CAUSES \\
\hline 119 & CARDIAC \\
\hline 120 & SIDE \\
\hline 121 & BECOME \\
\hline 122 & PROCEDURE \\
\hline 123 & DRUG \\
\hline
\end{tabular}




\begin{tabular}{|c|c|}
\hline 124 & DECREASED \\
\hline 125 & ACTION \\
\hline 126 & NEW \\
\hline 127 & DIFFERENT \\
\hline 128 & SURGICAL \\
\hline 129 & EFFECTIVE \\
\hline 130 & COMPLICATIONS \\
\hline 131 & ACTIVITIES \\
\hline 132 & FAILURE \\
\hline 133 & BRAIN \\
\hline 134 & MORAL \\
\hline 135 & MAKE \\
\hline 136 & SOCIAL \\
\hline 137 & TYPE \\
\hline 138 & PROBLEM \\
\hline 139 & WATER \\
\hline 140 & CONDITION \\
\hline 141 & CELL \\
\hline 142 & PRIMARY \\
\hline 143 & HISTORY \\
\hline 144 & POTENTIAL \\
\hline 145 & MEDICATION \\
\hline 146 & STAFF \\
\hline 147 & LIVER \\
\hline 148 & AREAS \\
\hline 149 & CHEST \\
\hline 150 & PRESCRIBED \\
\hline 151 & AVAILABLE \\
\hline 152 & MADE \\
\hline 153 & TAKE \\
\hline 154 & WEIGHT \\
\hline 155 & ILLNESS \\
\hline 156 & PRESENT \\
\hline 157 & DEVELOPMENT \\
\hline 158 & MAJOR \\
\hline 159 & DISORDERS \\
\hline 160 & ABILITY \\
\hline 161 & BED \\
\hline 162 & SEVERAL \\
\hline 163 & EXPERIENCE \\
\hline 164 & INTAKE \\
\hline 165 & REQUIRED \\
\hline 166 & BLEEDING \\
\hline
\end{tabular}

\begin{tabular}{|c|c|}
\hline 167 & GROUP \\
\hline 168 & LOWER \\
\hline 169 & AGENTS \\
\hline 170 & GROUPS \\
\hline 171 & ORAL \\
\hline 172 & STRESS \\
\hline 173 & EARLY \\
\hline 174 & ABLE \\
\hline 175 & FORM \\
\hline 176 & PLAN \\
\hline 177 & REDUCE \\
\hline 178 & NEEDED \\
\hline 179 & TEACHING \\
\hline 180 & ROLE \\
\hline 181 & DATA \\
\hline 182 & NERVE \\
\hline 183 & MEMBERS \\
\hline 184 & GENERAL \\
\hline 185 & PLACE \\
\hline 186 & AVOID \\
\hline 187 & PULMONARY \\
\hline 188 & OLDER \\
\hline 189 & WOUND \\
\hline 190 & DIAGNOSIS \\
\hline 191 & TUBE \\
\hline 192 & REQUIRE \\
\hline 193 & DIABETES \\
\hline 194 & SAME \\
\hline 195 & ASSESS \\
\hline 196 & LARGE \\
\hline 197 & GOALS \\
\hline 198 & UNIT \\
\hline 199 & CONDITIONS \\
\hline 200 & PERFORMED \\
\hline 201 & VOLUME \\
\hline 202 & DEATH \\
\hline 203 & LEFT \\
\hline 204 & OXYGEN \\
\hline 205 & EVEN \\
\hline 206 & HOSPITAL \\
\hline 207 & ETHICS \\
\hline 208 & CHILDREN \\
\hline 209 & ANOTHER \\
\hline
\end{tabular}

\begin{tabular}{|c|c|}
\hline 210 & DRAINAGE \\
\hline 211 & SERVICES \\
\hline 212 & CALLED \\
\hline 213 & SITE \\
\hline 214 & FREQUENTLY \\
\hline 215 & KNOWLEDGE \\
\hline 216 & PER \\
\hline 217 & INTERVENTIONS \\
\hline 218 & PERSONS \\
\hline 219 & RESEARCH \\
\hline 220 & URINARY \\
\hline 221 & ADDITION \\
\hline 222 & OWN \\
\hline 223 & FOOD \\
\hline 224 & BLADDER \\
\hline 225 & DAILY \\
\hline 226 & PERIOD \\
\hline 227 & BASED \\
\hline 228 & INDIVIDUALS \\
\hline 229 & EXERCISE \\
\hline 230 & IDENTIFY \\
\hline 231 & CAUSED \\
\hline 232 & OUTCOMES \\
\hline 233 & HUMAN \\
\hline 234 & ADEQUATE \\
\hline 235 & HEAD \\
\hline 236 & LOW \\
\hline 237 & ESPECIALLY \\
\hline 238 & PERSONAL \\
\hline 239 & GLUCOSE \\
\hline 240 & EFFECT \\
\hline 241 & INVOLVED \\
\hline 242 & DECREASE \\
\hline 243 & FLOW \\
\hline 244 & MUSCLES \\
\hline 245 & ETHICAL \\
\hline 246 & SEXUAL \\
\hline 247 & ADMINISTRATION \\
\hline 248 & ANXIETY \\
\hline 249 & EDUCATION \\
\hline 250 & MAINTAIN \\
\hline 251 & STATE \\
\hline 252 & VALUES \\
\hline
\end{tabular}




\begin{tabular}{|c|c|}
\hline 253 & AMOUNT \\
\hline 254 & TRACT \\
\hline 255 & DAMAGE \\
\hline 256 & FURTHER \\
\hline 257 & BACK \\
\hline 258 & CATHETER \\
\hline 259 & JOINT \\
\hline 260 & INCREASES \\
\hline 261 & HAND \\
\hline 262 & MEASURES \\
\hline 263 & COMMUNICATION \\
\hline 264 & PROCEDURES \\
\hline 265 & DETERMINE \\
\hline 266 & EXAMINATION \\
\hline 267 & TYPES \\
\hline 268 & PRO \\
\hline 269 & SKILLS \\
\hline 270 & SLEEP \\
\hline 271 & TEST \\
\hline 272 & EVERY \\
\hline 273 & DIET \\
\hline 274 & LUNG \\
\hline 275 & QUALITY \\
\hline 276 & TABLE \\
\hline 277 & EYE \\
\hline 278 & ENVIRONMENT \\
\hline 279 & ESSENTIAL \\
\hline 280 & ABDOMINAL \\
\hline 281 & COMMUNITY \\
\hline 282 & ARTERY \\
\hline 283 & LEARNING \\
\hline 284 & RESOURCES \\
\hline 285 & TAKEN \\
\hline 286 & CASES \\
\hline 287 & GOOD \\
\hline 288 & INFECTIONS \\
\hline 289 & KNOWN \\
\hline 290 & TEMPERATURE \\
\hline 291 & SIGNIFICANT \\
\hline 292 & INSULIN \\
\hline 293 & TISSUES \\
\hline 294 & WAY \\
\hline 295 & FOUND \\
\hline
\end{tabular}

\begin{tabular}{|c|c|}
\hline 296 & AFFECTED \\
\hline 297 & AIR \\
\hline 298 & IMPAIRED \\
\hline 299 & QUESTIONS \\
\hline 300 & MAKING \\
\hline 301 & SYSTEMS \\
\hline 302 & PLANNING \\
\hline 303 & STAGE \\
\hline 304 & INCLUDES \\
\hline 305 & MONITORING \\
\hline 306 & CASE \\
\hline 307 & ENSURE \\
\hline 308 & TEAM \\
\hline 309 & TREAT \\
\hline 310 & UPPER \\
\hline 311 & GREATER \\
\hline 312 & STUDY \\
\hline 313 & MOVEMENT \\
\hline 314 & SITUATION \\
\hline 315 & BOWEL \\
\hline 316 & LOCAL \\
\hline 317 & REQUIRES \\
\hline 318 & SERUM \\
\hline 319 & EVALUATION \\
\hline 320 & LEAD \\
\hline 321 & BREAST \\
\hline 322 & RATHER \\
\hline 323 & TIMES \\
\hline 324 & ELDERLY \\
\hline 325 & INCREASING \\
\hline 326 & ADULTS \\
\hline 327 & CONSIDERED \\
\hline 328 & BECOMES \\
\hline 329 & INVOLVES \\
\hline 330 & ISSUES \\
\hline 331 & LIKELY \\
\hline 332 & ADMINISTERED \\
\hline 333 & CONTACT \\
\hline 334 & DIAGNOSTIC \\
\hline 335 & STATES \\
\hline 336 & CERTAIN \\
\hline 337 & VESSELS \\
\hline 338 & PROVIDES \\
\hline
\end{tabular}

\begin{tabular}{|c|c|}
\hline 339 & MEN \\
\hline 340 & EVIDENCE \\
\hline 341 & VENOUS \\
\hline 342 & ALCOHOL \\
\hline 343 & OUTPUT \\
\hline 344 & AGAINST \\
\hline 345 & GROWTH \\
\hline 346 & PARTICULARLY \\
\hline 347 & MEANS \\
\hline 348 & PUBLIC \\
\hline 349 & VARIOUS \\
\hline 350 & REST \\
\hline 351 & POSITIVE \\
\hline 352 & DISORDER \\
\hline 353 & PRESENCE \\
\hline 354 & STRATEGIES \\
\hline 355 & REDUCED \\
\hline 356 & LONG \\
\hline 357 & PULSE \\
\hline 358 & EMOTIONAL \\
\hline 359 & SODIUM \\
\hline 360 & COMMONLY \\
\hline 361 & PROMOTE \\
\hline 362 & TRAUMA \\
\hline 363 & DIFFICULT \\
\hline 364 & SPINAL \\
\hline 365 & AFFECT \\
\hline 366 & DECISIONS \\
\hline 367 & TREATED \\
\hline 368 & ASSIST \\
\hline 369 & MONTHS \\
\hline 370 & PROFESSIONAL \\
\hline 371 & PARTICULAR \\
\hline 372 & SHOCK \\
\hline 373 & EDEMA \\
\hline 374 & FLUIDS \\
\hline 375 & CHART \\
\hline 376 & RESPONSIBILITY \\
\hline 377 & MENTAL \\
\hline 378 & ARTERIAL \\
\hline 379 & TESTS \\
\hline 380 & RIGHTS \\
\hline 381 & CARING \\
\hline
\end{tabular}




\begin{tabular}{|c|c|}
\hline 382 & MOUTH \\
\hline 383 & RESULTING \\
\hline 384 & ACTIONS \\
\hline 385 & FINDINGS \\
\hline 386 & GASTRIC \\
\hline 387 & LONGTERM \\
\hline 388 & REMOVED \\
\hline 389 & DISEASES \\
\hline 390 & SELFCARE \\
\hline 391 & PROTEIN \\
\hline 392 & SURFACE \\
\hline 393 & HEALTHCARE \\
\hline 394 & PRODUCE \\
\hline 395 & SYNDROME \\
\hline 396 & APPROACH \\
\hline 397 & BALANCE \\
\hline 398 & METHODS \\
\hline 399 & MIGHT \\
\hline 400 & EXCESSIVE \\
\hline 401 & PROVIDING \\
\hline 402 & PROVIDED \\
\hline 403 & REPORT \\
\hline 404 & ACTIVE \\
\hline 405 & COULD \\
\hline 406 & UNDERSTANDING \\
\hline 407 & PREVENTION \\
\hline 408 & HORMONE \\
\hline 409 & BREATHING \\
\hline 410 & RANGE \\
\hline 411 & MAIN \\
\hline 412 & END \\
\hline 413 & FEW \\
\hline 414 & DOSE \\
\hline 415 & SENSORY \\
\hline 416 & TAKING \\
\hline 417 & BEHAVIOR \\
\hline 418 & FEVER \\
\hline 419 & HEALING \\
\hline 420 & STUDIES \\
\hline 421 & ACID \\
\hline 422 & GIVE \\
\hline 423 & SECONDARY \\
\hline 424 & LESIONS \\
\hline
\end{tabular}

\begin{tabular}{|c|c|}
\hline 425 & CRITICAL \\
\hline 426 & PERFORMANCE \\
\hline 427 & POSTOPERATIVE \\
\hline 428 & DOWN \\
\hline 429 & PHASE \\
\hline 430 & FOCUS \\
\hline 431 & OPEN \\
\hline 432 & BEST \\
\hline 433 & DISCHARGE \\
\hline 434 & HAIR \\
\hline 435 & USEFUL \\
\hline 436 & REGULAR \\
\hline 437 & EXPOSURE \\
\hline 438 & FEELINGS \\
\hline 439 & INFLAMMATION \\
\hline 440 & FORMATION \\
\hline 441 & TOTAL \\
\hline 442 & ASSESSED \\
\hline 443 & SENSE \\
\hline 444 & SIMILAR \\
\hline 445 & WALL \\
\hline 446 & ORDER \\
\hline 447 & RADIATION \\
\hline 448 & NUTRITIONAL \\
\hline 449 & EXTERNAL \\
\hline 450 & SAFETY \\
\hline 451 & EQUIPMENT \\
\hline 452 & PERIPHERAL \\
\hline 453 & SUPPLY \\
\hline 454 & CALCIUM \\
\hline 455 & AMONG \\
\hline 456 & COMPLETE \\
\hline 457 & LEAST \\
\hline 458 & METHOD \\
\hline 459 & VASCULAR \\
\hline 460 & CAUSING \\
\hline 461 & SEEN \\
\hline 462 & DESCRIBE \\
\hline 463 & HEARING \\
\hline 464 & SIZE \\
\hline 465 & LATER \\
\hline 466 & CIRCULATION \\
\hline 467 & MOTOR \\
\hline
\end{tabular}

\begin{tabular}{|c|c|}
\hline 468 & ENERGY \\
\hline 469 & $\mathrm{ACT}$ \\
\hline 470 & CORD \\
\hline 471 & EXPECTED \\
\hline 472 & MOVE \\
\hline 473 & VITAL \\
\hline 474 & DESCRIBED \\
\hline 475 & NATURE \\
\hline 476 & IMPROVE \\
\hline 477 & RESPONSES \\
\hline 478 & ABOVE \\
\hline 479 & DIRECT \\
\hline 480 & DISCOMFORT \\
\hline 481 & ABNORMAL \\
\hline 482 & RESPONSIBLE \\
\hline 483 & CENTRAL \\
\hline 484 & HAVING \\
\hline 485 & KIDNEY \\
\hline 486 & PLACED \\
\hline 487 & AROUND \\
\hline 488 & HIGHER \\
\hline 489 & VOMITING \\
\hline 490 & FOODS \\
\hline 491 & FREQUENT \\
\hline 492 & GLAND \\
\hline 493 & DECISION \\
\hline 494 & PRODUCTION \\
\hline 495 & LACK \\
\hline 496 & OBSTRUCTION \\
\hline 497 & MANAGER \\
\hline 498 & DEGREE \\
\hline 499 & IMMUNE \\
\hline 500 & SERIOUS \\
\hline 501 & PLASMA \\
\hline 502 & DEPRESSION \\
\hline 503 & IDENTIFIED \\
\hline 504 & VEIN \\
\hline 505 & AGENT \\
\hline 506 & TERMS \\
\hline 507 & FEEL \\
\hline 508 & CEREBRAL \\
\hline 509 & COPING \\
\hline 510 & INDICATED \\
\hline
\end{tabular}




\begin{tabular}{|c|c|}
\hline 511 & KNOW \\
\hline 512 & TUMOR \\
\hline 513 & LONGER \\
\hline 514 & ONCE \\
\hline 515 & VISION \\
\hline 516 & INTERVENTION \\
\hline 517 & APPLIED \\
\hline 518 & NECK \\
\hline 519 & RED \\
\hline 520 & LUNGS \\
\hline 521 & MONITOR \\
\hline 522 & CAVITY \\
\hline 523 & PRINCIPLES \\
\hline 524 & FORMS \\
\hline 525 & SPECIAL \\
\hline 526 & HYPERTENSION \\
\hline 527 & INCIDENCE \\
\hline 528 & NORMALLY \\
\hline 529 & REACTION \\
\hline 530 & REFERRED \\
\hline 531 & SET \\
\hline 532 & THYROID \\
\hline 533 & EXERCISES \\
\hline 534 & RAPID \\
\hline 535 & FAMILIES \\
\hline 536 & GENERALLY \\
\hline 537 & THEORY \\
\hline 538 & COMPLEX \\
\hline 539 & NUTRITION \\
\hline 540 & PHYSICIAN \\
\hline 541 & THERAPEUTIC \\
\hline 542 & ANTERIOR \\
\hline 543 & STRUCTURES \\
\hline 544 & MANIFESTATIONS \\
\hline 545 & NOW \\
\hline 546 & PROCESSES \\
\hline 547 & INTERNAL \\
\hline 548 & IMPORTANCE \\
\hline 549 & ACCESS \\
\hline 550 & FACTOR \\
\hline 551 & SETTING \\
\hline 552 & EXAMPLES \\
\hline 553 & PERFORM \\
\hline
\end{tabular}

\begin{tabular}{|c|c|}
\hline 554 & DEEP \\
\hline 555 & REMOVAL \\
\hline 556 & POWER \\
\hline $\mathbf{5 5 7}$ & BELOW \\
\hline 558 & MINUTES \\
\hline 559 & NERVES \\
\hline 560 & AIRWAY \\
\hline 561 & FATIGUE \\
\hline 562 & EAR \\
\hline 563 & LIGHT \\
\hline 564 & ADDITIONAL \\
\hline 565 & NAUSEA \\
\hline 566 & GOAL \\
\hline 567 & INITIAL \\
\hline 568 & SITUATIONS \\
\hline 569 & POOR \\
\hline 570 & VARIETY \\
\hline 571 & HORMONES \\
\hline 572 & INDICATE \\
\hline 573 & ALWAYS \\
\hline 574 & DISCUSSED \\
\hline 575 & HELPS \\
\hline 576 & REPORTED \\
\hline 577 & STRUCTURE \\
\hline 578 & FUNCTIONS \\
\hline 579 & PAST \\
\hline 580 & UNDERSTAND \\
\hline 581 & CONSIDER \\
\hline 582 & SECRETIONS \\
\hline 583 & YEAR \\
\hline 584 & MEMBRANE \\
\hline 585 & NERVOUS \\
\hline 586 & DRY \\
\hline $\mathbf{5 8 7}$ & HEALTHY \\
\hline 588 & CURRENT \\
\hline 589 & POINT \\
\hline 590 & SOLUTION \\
\hline 591 & SOMETIMES \\
\hline 592 & METABOLIC \\
\hline 593 & TECHNIQUES \\
\hline 594 & ACCORDING \\
\hline 595 & ACHIEVE \\
\hline 596 & CULTURAL \\
\hline
\end{tabular}

\begin{tabular}{|c|c|}
\hline 597 & PRACTICES \\
\hline 598 & STOMACH \\
\hline 599 & ATTENTION \\
\hline 600 & PROLONGED \\
\hline 601 & BASIS \\
\hline 602 & KEY \\
\hline 603 & LIKE \\
\hline 604 & LYMPH \\
\hline 605 & ONSET \\
\hline 606 & COURSE \\
\hline 607 & KEEP \\
\hline 608 & DRESSING \\
\hline 609 & MANAGE \\
\hline 610 & FACE \\
\hline 611 & WAYS \\
\hline 612 & STERILE \\
\hline 613 & SPACE \\
\hline 614 & ASSISTANCE \\
\hline 615 & EMERGENCY \\
\hline 616 & ASK \\
\hline 617 & DISCUSS \\
\hline 618 & LIFESTYLE \\
\hline 619 & MONITORED \\
\hline 620 & RELATIONSHIP \\
\hline 621 & CONCERNS \\
\hline 622 & NEGATIVE \\
\hline 623 & SOUNDS \\
\hline 624 & ALLOW \\
\hline 625 & CLOSE \\
\hline 626 & CHILD \\
\hline 627 & TESTING \\
\hline 628 & CLEAR \\
\hline 629 & NATIONAL \\
\hline 630 & OBTAINED \\
\hline 631 & PSYCHOLOGICAL \\
\hline 632 & SECRETION \\
\hline 633 & SECOND \\
\hline 634 & AWARE \\
\hline 635 & VISUAL \\
\hline 636 & DIETARY \\
\hline 637 & PREGNANCY \\
\hline 638 & SYSTEMIC \\
\hline 639 & BOX \\
\hline
\end{tabular}




\begin{tabular}{|c|c|}
\hline 640 & DEVICES \\
\hline 641 & RELEASE \\
\hline 642 & DIAGNOSES \\
\hline 643 & REACTIONS \\
\hline 644 & DEVELOPED \\
\hline 645 & LIVING \\
\hline 646 & MULTIPLE \\
\hline 647 & BELIEFS \\
\hline 648 & GASTROINTESTINAL \\
\hline 649 & ILL \\
\hline 650 & ABSENCE \\
\hline 651 & ORGANS \\
\hline 652 & APPEAR \\
\hline 653 & BONES \\
\hline 654 & GLANDS \\
\hline 655 & POSTERIOR \\
\hline 656 & DIFFICULTY \\
\hline 657 & SHORT \\
\hline 658 & WORKING \\
\hline 659 & POTASSIUM \\
\hline 660 & POLICY \\
\hline 661 & SMOKING \\
\hline 662 & FOOT \\
\hline 663 & LEADING \\
\hline 664 & ORGANIZATION \\
\hline 665 & SOCIETY \\
\hline 666 & IMMEDIATELY \\
\hline 667 & RETURN \\
\hline 668 & SPREAD \\
\hline 669 & VIEW \\
\hline 670 & GAS \\
\hline 671 & ADULT \\
\hline 672 & VENTRICULAR \\
\hline 673 & JUST \\
\hline 674 & LEARN \\
\hline 675 & ALTERED \\
\hline 676 & VEINS \\
\hline 677 & REMOVE \\
\hline 678 & CARDIOVASCULAR \\
\hline 679 & RELIEF \\
\hline 680 & FEAR \\
\hline 681 & COUGH \\
\hline 682 & FOLLOW \\
\hline
\end{tabular}

\begin{tabular}{|c|c|}
\hline 683 & DISCUSSION \\
\hline 684 & ENCOURAGED \\
\hline 685 & CULTURE \\
\hline 686 & PREPARATION \\
\hline 687 & SUBSTANCES \\
\hline 688 & SITES \\
\hline 689 & DOSES \\
\hline 690 & JOB \\
\hline 691 & SHOWN \\
\hline 692 & PREVIOUS \\
\hline 693 & PROFESSIONALS \\
\hline 694 & STROKE \\
\hline 695 & BREATH \\
\hline 696 & GENETIC \\
\hline 697 & STAGES \\
\hline 698 & DEFICIENCY \\
\hline 699 & MANAGING \\
\hline 700 & PROMOTION \\
\hline 701 & PROVIDERS \\
\hline 702 & RECOMMENDED \\
\hline 703 & RECORD \\
\hline 704 & UNITED \\
\hline 705 & ARM \\
\hline 706 & STANDARDS \\
\hline 707 & UNITS \\
\hline 708 & ADVERSE \\
\hline 709 & INJURIES \\
\hline 710 & NOTE \\
\hline 711 & VITAMIN \\
\hline 712 & STILL \\
\hline 713 & TREATMENTS \\
\hline 714 & HELPFUL \\
\hline 715 & SOUND \\
\hline 716 & MODEL \\
\hline 717 & ROOM \\
\hline 718 & RESISTANCE \\
\hline 719 & PROMOTING \\
\hline 720 & CHARACTERISTICS \\
\hline 721 & DEVELOPS \\
\hline 722 & MANAGERS \\
\hline 723 & PATTERN \\
\hline 724 & DIRECTLY \\
\hline 725 & PROGRAMS \\
\hline
\end{tabular}

\begin{tabular}{|c|c|}
\hline 726 & TECHNIQUE \\
\hline 727 & FRACTURE \\
\hline 728 & INSERTED \\
\hline 729 & BETTER \\
\hline 730 & FAT \\
\hline 731 & FOUR \\
\hline 732 & PREVENTING \\
\hline 733 & TAKES \\
\hline 734 & MEET \\
\hline 735 & OBTAIN \\
\hline 736 & ALONG \\
\hline 737 & ELEVATED \\
\hline 738 & INFLUENCE \\
\hline 739 & REMAIN \\
\hline 740 & ARTERIES \\
\hline 741 & STRENGTH \\
\hline 742 & EXTENT \\
\hline 743 & DEPENDS \\
\hline 744 & ROLES \\
\hline 745 & EXPLAIN \\
\hline 746 & PRODUCTS \\
\hline 747 & FUTURE \\
\hline 748 & PROGRAM \\
\hline 749 & PURPOSE \\
\hline 750 & SOURCE \\
\hline 751 & CHEMOTHERAPY \\
\hline 752 & DEVELOPING \\
\hline 753 & IMMEDIATE \\
\hline 754 & COMFORT \\
\hline 755 & CONTINUOUS \\
\hline 756 & ONES \\
\hline 757 & SWELLING \\
\hline 758 & ALLOWS \\
\hline 759 & CONCENTRATION \\
\hline 760 & YOUNG \\
\hline 761 & HYGIENE \\
\hline 762 & INFORMED \\
\hline 763 & ORGANIZATIONS \\
\hline 764 & LEG \\
\hline 765 & RECEIVE \\
\hline 766 & DISABILITY \\
\hline 767 & FULL \\
\hline 768 & SCREENING \\
\hline
\end{tabular}




\begin{tabular}{|c|c|}
\hline 769 & APPROXIMATELY \\
\hline 770 & BEHAVIORS \\
\hline 771 & TUMORS \\
\hline 772 & UNDERLYING \\
\hline 773 & VALUE \\
\hline 774 & SERVICE \\
\hline 775 & PARTS \\
\hline 776 & WEAKNESS \\
\hline 777 & REDUCING \\
\hline 778 & EXPERIENCES \\
\hline 779 & MAINTAINING \\
\hline 780 & ORGAN \\
\hline 781 & SURE \\
\hline 782 & ELECTROLYTE \\
\hline 783 & SEX \\
\hline 784 & INFLAMMATORY \\
\hline 785 & MILD \\
\hline 786 & VARY \\
\hline 787 & PROVIDER \\
\hline 788 & JOINTS \\
\hline 789 & STIMULATION \\
\hline 790 & FREE \\
\hline 791 & RATIONALE \\
\hline 792 & REDUCTION \\
\hline 793 & CHEMICAL \\
\hline 794 & CONTINUE \\
\hline 795 & COUNT \\
\hline 796 & MOBILITY \\
\hline 797 & PROGRESS \\
\hline 798 & THEMSELVES \\
\hline 799 & GUIDELINES \\
\hline 800 & POPULATION \\
\hline 801 & DYSFUNCTION \\
\hline 802 & ENCOURAGE \\
\hline 803 & PERIODS \\
\hline 804 & FUNCTIONING \\
\hline 805 & HANDS \\
\hline 806 & RELATIONSHIPS \\
\hline 807 & ASPECTS \\
\hline 808 & THOUGHT \\
\hline 809 & MARROW \\
\hline 810 & DEPENDING \\
\hline 811 & FUNCTIONAL \\
\hline
\end{tabular}

\begin{tabular}{|c|c|}
\hline 812 & REGIMEN \\
\hline 813 & RESPOND \\
\hline 814 & FIND \\
\hline 815 & BILE \\
\hline 816 & LIMITED \\
\hline 817 & AGENCY \\
\hline 818 & ADVANCED \\
\hline 819 & FOLLOWUP \\
\hline 820 & HEAT \\
\hline 821 & APPLY \\
\hline 822 & EVALUATE \\
\hline 823 & MALIGNANT \\
\hline 824 & PRODUCED \\
\hline 825 & TOGETHER \\
\hline 826 & TOWARD \\
\hline 827 & NEXT \\
\hline 828 & USES \\
\hline 829 & RAPIDLY \\
\hline 830 & RELIEVE \\
\hline 831 & RECOVERY \\
\hline 832 & MAN \\
\hline 833 & PROTECT \\
\hline 834 & ABUSE \\
\hline 835 & REVIEW \\
\hline 836 & LINE \\
\hline 837 & KIDNEYS \\
\hline 838 & RECEIVING \\
\hline 839 & LEADS \\
\hline 840 & VENTILATION \\
\hline 841 & AIDS \\
\hline 842 & LOCATION \\
\hline 843 & SAFE \\
\hline 844 & BIOPSY \\
\hline 845 & ASSESSING \\
\hline 846 & LABORATORY \\
\hline 847 & WOMAN \\
\hline 848 & MASS \\
\hline 849 & WORLD \\
\hline 850 & ALERT \\
\hline 851 & AMOUNTS \\
\hline 852 & AVOIDED \\
\hline 853 & SETTINGS \\
\hline 854 & VALVE \\
\hline
\end{tabular}

\begin{tabular}{|c|c|}
\hline 855 & FREQUENCY \\
\hline 856 & NEUROLOGIC \\
\hline 857 & CORRECT \\
\hline 858 & ABDOMEN \\
\hline 859 & CONCERN \\
\hline 860 & INTESTINAL \\
\hline 861 & INVOLVE \\
\hline 862 & CONSISTS \\
\hline 863 & USUAL \\
\hline 864 & PATTERNS \\
\hline 865 & SOFT \\
\hline 866 & CORONARY \\
\hline 867 & DURATION \\
\hline 868 & REMAINS \\
\hline 869 & SEVERITY \\
\hline 870 & APPEARANCE \\
\hline 871 & BEGIN \\
\hline 872 & TEND \\
\hline 873 & BEGINS \\
\hline 874 & FORMED \\
\hline 875 & FRACTURES \\
\hline 876 & NASAL \\
\hline 877 & EMPLOYEES \\
\hline 878 & GRADUALLY \\
\hline 879 & INTRAVENOUS \\
\hline 880 & RECENT \\
\hline 881 & SPIRITUAL \\
\hline 882 & DEPARTMENT \\
\hline 883 & CONFLICT \\
\hline 884 & DEVICE \\
\hline 885 & IMPULSES \\
\hline 886 & REPLACEMENT \\
\hline 887 & RETENTION \\
\hline 888 & INCISION \\
\hline 889 & SELF \\
\hline 890 & EXPERIENCED \\
\hline 891 & IMPACT \\
\hline 892 & INADEQUATE \\
\hline 893 & ALTERNATIVE \\
\hline 894 & DIE \\
\hline 895 & COLOR \\
\hline 896 & CONTINUED \\
\hline 897 & LITTLE \\
\hline
\end{tabular}




\begin{tabular}{|c|c|}
\hline 898 & CERVICAL \\
\hline 899 & EFFECTIVENESS \\
\hline 900 & INJECTION \\
\hline 901 & TERM \\
\hline 902 & MIDDLE \\
\hline 903 & PAINFUL \\
\hline 904 & MALE \\
\hline 905 & INTESTINE \\
\hline 906 & ULCERS \\
\hline 907 & ABSORPTION \\
\hline 908 & CONTROLLED \\
\hline 909 & DISTRESS \\
\hline 910 & FOLLOWED \\
\hline 911 & MAINTENANCE \\
\hline 912 & PARENTS \\
\hline 913 & THINKING \\
\hline 914 & EVENTS \\
\hline 915 & CARRIED \\
\hline 916 & IMAGE \\
\hline 917 & REPORTS \\
\hline 918 & HEADACHE \\
\hline 919 & PHYSIOLOGIC \\
\hline 920 & SUBSTANCE \\
\hline 921 & INTEGRITY \\
\hline 922 & HEPATITIS \\
\hline 923 & QUICKLY \\
\hline 924 & MECHANICAL \\
\hline 925 & OUTCOME \\
\hline 926 & WRITTEN \\
\hline 927 & BASIC \\
\hline 928 & CHOICE \\
\hline 929 & MYOCARDIAL \\
\hline 930 & DETERMINED \\
\hline 931 & INFUSION \\
\hline 932 & ANALYSIS \\
\hline 933 & HOUR \\
\hline 934 & EXCESS \\
\hline 935 & EYES \\
\hline 936 & MAKES \\
\hline 937 & COLD \\
\hline 938 & DECREASES \\
\hline 939 & REGARDING \\
\hline 940 & STANDARD \\
\hline
\end{tabular}

\begin{tabular}{|c|c|}
\hline 941 & EASILY \\
\hline 942 & FEET \\
\hline 943 & REHABILITATION \\
\hline 944 & RISKS \\
\hline 945 & LAW \\
\hline 946 & MOVEMENTS \\
\hline 947 & MUCOUS \\
\hline 948 & REFLEX \\
\hline 949 & SIMPLE \\
\hline 950 & UNABLE \\
\hline 951 & COGNITIVE \\
\hline 952 & MEASURE \\
\hline 953 & VAGINAL \\
\hline 954 & ASSESSES \\
\hline 955 & CLOSELY \\
\hline 956 & DELIVERY \\
\hline 957 & PELVIC \\
\hline 958 & MECHANISMS \\
\hline 959 & HOSPITALS \\
\hline 960 & IMPAIRMENT \\
\hline 961 & TRAINING \\
\hline 962 & CAREFULLY \\
\hline 963 & TASK \\
\hline 964 & WHITE \\
\hline 965 & GREAT \\
\hline 966 & MUCOSA \\
\hline 967 & PRECAUTIONS \\
\hline 968 & PRODUCES \\
\hline 969 & LAST \\
\hline 970 & CAPACITY \\
\hline 971 & BACTERIA \\
\hline 972 & COMBINATION \\
\hline 973 & EXCHANGE \\
\hline 974 & PITUITARY \\
\hline 975 & ANTIBIOTICS \\
\hline 976 & BASE \\
\hline 977 & ESTABLISHED \\
\hline 978 & NEEDLE \\
\hline 979 & NODES \\
\hline 980 & SOURCES \\
\hline 981 & CRISIS \\
\hline 982 & DEMONSTRATE \\
\hline 983 & MATERIAL \\
\hline
\end{tabular}

\begin{tabular}{|c|c|}
\hline 984 & PROPER \\
\hline 985 & TRANSMISSION \\
\hline 986 & COLON \\
\hline 987 & ENDOCRINE \\
\hline 988 & FEMALE \\
\hline 989 & SECTION \\
\hline 990 & ACHIEVED \\
\hline 991 & BENEFITS \\
\hline 992 & CANAL \\
\hline 993 & DESIRED \\
\hline 994 & GET \\
\hline 995 & NATURAL \\
\hline 996 & VIRUS \\
\hline 997 & AGING \\
\hline 998 & CAREFUL \\
\hline 999 & METABOLISM \\
\hline 1000 & OBSERVED \\
\hline 1001 & PREOPERATIVE \\
\hline 1002 & CLEAN \\
\hline 1003 & FIBRES \\
\hline 1004 & INCONTINENCE \\
\hline 1005 & INTER \\
\hline 1006 & REQUIREMENTS \\
\hline 1007 & RESPECT \\
\hline 1008 & CONTRACTION \\
\hline 1009 & NOTED \\
\hline 1010 & SENSATION \\
\hline 1011 & SENSITIVE \\
\hline 1012 & SUDDEN \\
\hline 1013 & ASPIRATION \\
\hline 1014 & CONCERNED \\
\hline 1015 & DEAL \\
\hline 1016 & WHOLE \\
\hline 1017 & COME \\
\hline 1018 & EFFORT \\
\hline 1019 & PROTECTION \\
\hline 1020 & CONTRAST \\
\hline 1021 & INSTRUCTED \\
\hline 1022 & LATERAL \\
\hline 1023 & LOST \\
\hline 1024 & ASSOCIATION \\
\hline 1025 & BACTERIAL \\
\hline 1026 & CHECK \\
\hline
\end{tabular}




\begin{tabular}{|c|c|}
\hline 1027 & LENGTH \\
\hline 1028 & PNEUMONIA \\
\hline 1029 & PREVIOUSLY \\
\hline 1030 & ROUTINE \\
\hline 1031 & ULCER \\
\hline 1032 & EATING \\
\hline 1033 & IRON \\
\hline 1034 & AFFECTS \\
\hline 1035 & SHOW \\
\hline 1036 & ANEMIA \\
\hline 1037 & TAUGHT \\
\hline 1038 & HIGHLY \\
\hline 1039 & LEGAL \\
\hline 1040 & MEMBER \\
\hline 1041 & NIGHT \\
\hline 1042 & APPEARS \\
\hline 1043 & DISABILITIES \\
\hline 1044 & MINIMIZE \\
\hline 1045 & RULES \\
\hline 1046 & CYCLE \\
\hline 1047 & FEELING \\
\hline 1048 & ACCURATE \\
\hline 1049 & CONCEPTS \\
\hline 1050 & GIVING \\
\hline 1051 & WELLBEING \\
\hline 1052 & SEIZURES \\
\hline 1053 & SUCTION \\
\hline 1054 & THEORIES \\
\hline 1055 & PRIOR \\
\hline 1056 & CHANGING \\
\hline 1057 & ASKED \\
\hline 1058 & HEMORRHAGE \\
\hline 1059 & MEANING \\
\hline 1060 & CONTAIN \\
\hline 1061 & DEMANDS \\
\hline 1062 & ONGOING \\
\hline 1063 & TURN \\
\hline 1064 & SOON \\
\hline 1065 & REASONS \\
\hline 1066 & ANTIBODIES \\
\hline 1067 & INFECTED \\
\hline 1068 & LIVE \\
\hline 1069 & SIGN \\
\hline
\end{tabular}

\begin{tabular}{|c|c|}
\hline 1070 & SLOWLY \\
\hline 1071 & ANALGESIC \\
\hline 1072 & CONTAINS \\
\hline 1073 & PROGRESSIVE \\
\hline 1074 & CONTRIBUTE \\
\hline 1075 & LEADERSHIP \\
\hline 1076 & ADRENAL \\
\hline 1077 & ALONE \\
\hline 1078 & EMPLOYEE \\
\hline 1079 & INEFFECTIVE \\
\hline 1080 & ELECTRICAL \\
\hline 1081 & ELIMINATION \\
\hline 1082 & SINGLE \\
\hline 1083 & SLOW \\
\hline 1084 & PERFUSION \\
\hline 1085 & CIRCUMSTANCES \\
\hline 1086 & DUCT \\
\hline 1087 & EFFECTIVELY \\
\hline 1088 & RAISED \\
\hline 1089 & APPROACHES \\
\hline 1090 & DEPENDENT \\
\hline 1091 & IMPROVED \\
\hline 1092 & LAYER \\
\hline 1093 & PROSTATE \\
\hline 1094 & NOSE \\
\hline 1095 & PERSONNEL \\
\hline 1096 & BREAKDOWN \\
\hline 1097 & RELATIVELY \\
\hline 1098 & NAME \\
\hline 1099 & PLANS \\
\hline 1100 & ELDERS \\
\hline 1101 & FULLY \\
\hline 1102 & LIVES \\
\hline 1103 & PLEURAL \\
\hline 1104 & STUDENTS \\
\hline 1105 & VENTRICLE \\
\hline 1106 & AUTHORITY \\
\hline 1107 & CLOSED \\
\hline 1108 & CONSEQUENCES \\
\hline 1109 & INDICATES \\
\hline 1110 & IRRITATION \\
\hline 1111 & XRAY \\
\hline 1112 & CONSTIPATION \\
\hline
\end{tabular}

1113 EXTREMITIES

\begin{tabular}{|c|c|}
\hline 1114 & MEASURED \\
\hline 1115 & REASON \\
\hline 1116 & RESPONSIBILITIES \\
\hline 1117 & SMOOTH \\
\hline 1118 & SPEECH \\
\hline 1119 & BENEFIT \\
\hline 1120 & CONSENT \\
\hline 1121 & DETECT \\
\hline 1122 & DISTAL \\
\hline 1123 & GAIN \\
\hline 1124 & ORGANISMS \\
\hline 1125 & COMPRESSION \\
\hline 1126 & RELIGIOUS \\
\hline 1127 & SEEK \\
\hline 1128 & VIRAL \\
\hline 1129 & ACCOUNT \\
\hline 1130 & DIALYSIS \\
\hline 1131 & INFECTIOUS \\
\hline 1132 & INSTRUCTIONS \\
\hline 1133 & REDUCES \\
\hline 1134 & SKILL \\
\hline 1135 & IMPROVEMENT \\
\hline 1136 & MEALS \\
\hline 1137 & TEETH \\
\hline 1138 & ENOUGH \\
\hline 1139 & LEGS \\
\hline 1140 & LESION \\
\hline 1141 & PLAY \\
\hline 1142 & PROTEINS \\
\hline 1143 & TOLERANCE \\
\hline 1144 & CHARACTERIZED \\
\hline 1145 & PUT \\
\hline 1146 & THERAPIES \\
\hline 1147 & ANESTHESIA \\
\hline 1148 & AWAY \\
\hline 1149 & CENTER \\
\hline 1150 & FACILITATE \\
\hline 1151 & TASKS \\
\hline 1152 & DIFFERENCES \\
\hline 1153 & PERCEPTION \\
\hline 1154 & RELEVANT \\
\hline 1155 & TUBES \\
\hline
\end{tabular}




\begin{tabular}{|c|c|}
\hline 1156 & EXTREMITY \\
\hline 1157 & FACT \\
\hline 1158 & HERE \\
\hline 1159 & ACTUAL \\
\hline 1160 & CONTINUES \\
\hline 1161 & FOREIGN \\
\hline 1162 & TOUCH \\
\hline 1163 & THORACIC \\
\hline 1164 & CARRY \\
\hline 1165 & WORDS \\
\hline 1166 & AGENCIES \\
\hline 1167 & ENVIRONMENTAL \\
\hline 1168 & MAINTAINED \\
\hline 1169 & NEVER \\
\hline 1170 & PRACTICAL \\
\hline 1171 & RATES \\
\hline 1172 & COMPONENTS \\
\hline 1173 & COSTS \\
\hline 1174 & MEDICINE \\
\hline 1175 & PLATELET \\
\hline 1176 & RELATION \\
\hline 1177 & ANTIBIOTIC \\
\hline 1178 & BUM \\
\hline 1179 & DEFINED \\
\hline 1180 & REPEATED \\
\hline 1181 & STOOL \\
\hline 1182 & TENSION \\
\hline 1183 & TONGUE \\
\hline 1184 & ITSELF \\
\hline 1185 & TUMOUR \\
\hline 1186 & DIRECTED \\
\hline 1187 & HIP \\
\hline 1188 & LIMB \\
\hline 1189 & SINCE \\
\hline 1190 & STRONG \\
\hline 1191 & ALLERGIC \\
\hline 1192 & MOTION \\
\hline 1193 & RECORDED \\
\hline 1194 & ACROSS \\
\hline 1195 & DEHYDRATION \\
\hline 1196 & EXTENSIVE \\
\hline 1197 & PSYCHOSOCIAL \\
\hline 1198 & SUCCESSFUL \\
\hline
\end{tabular}

\begin{tabular}{|c|c|}
\hline 1199 & WARM \\
\hline 1200 & AWARENESS \\
\hline 1201 & CHANGED \\
\hline 1202 & MATERIALS \\
\hline 1203 & MEMBRANES \\
\hline 1204 & PERMANENT \\
\hline 1205 & QUESTION \\
\hline 1206 & FEEDING \\
\hline 1207 & JUSTICE \\
\hline 1208 & OEDEMA \\
\hline 1209 & ESTABLISH \\
\hline 1210 & RECOGNIZE \\
\hline 1211 & WOUNDS \\
\hline 1212 & DUTY \\
\hline 1213 & EVENT \\
\hline 1214 & MECHANISM \\
\hline 1215 & RELAXATION \\
\hline 1216 & SOLUTIONS \\
\hline 1217 & APPLICATION \\
\hline 1218 & CELLULAR \\
\hline 1219 & CONTINUING \\
\hline 1220 & COPE \\
\hline 1221 & IDENTIFYING \\
\hline 1222 & INSERTION \\
\hline 1223 & PARTIAL \\
\hline 1224 & WALLS \\
\hline 1225 & ATRIAL \\
\hline 1226 & COUGHING \\
\hline 1227 & FORCE \\
\hline 1228 & SURGEON \\
\hline 1229 & VARIES \\
\hline 1230 & CONTENT \\
\hline 1231 & OVERALL \\
\hline 1232 & PREVENTS \\
\hline 1233 & KEPT \\
\hline 1234 & RHYTHM \\
\hline 1235 & DETERMINING \\
\hline 1236 & OUTSIDE \\
\hline 1237 & PORTION \\
\hline 1238 & SPECIMEN \\
\hline 1239 & SURVIVAL \\
\hline 1240 & ADVISED \\
\hline 1241 & ARISE \\
\hline
\end{tabular}

\begin{tabular}{|c|c|}
\hline 1242 & COST \\
\hline 1243 & INTERVIEW \\
\hline 1244 & OPERATION \\
\hline 1245 & NESS \\
\hline 1246 & OFFER \\
\hline 1247 & TUMOURS \\
\hline 1248 & ADDRESS \\
\hline 1249 & EVENTUALLY \\
\hline 1250 & FACILITIES \\
\hline 1251 & ATTEMPT \\
\hline 1252 & DRESSINGS \\
\hline 1253 & FIVE \\
\hline 1254 & IMPLICATIONS \\
\hline 1255 & LIST \\
\hline 1256 & POLICIES \\
\hline 1257 & COMPLETELY \\
\hline 1258 & CONFUSION \\
\hline 1259 & GLOVES \\
\hline 1260 & LANGUAGE \\
\hline 1261 & OPPORTUNITY \\
\hline 1262 & RARELY \\
\hline 1263 & WIDE \\
\hline 1264 & DECISIONMAKING \\
\hline 1265 & INITIALLY \\
\hline 1266 & MOVING \\
\hline 1267 & PARTICIPATE \\
\hline 1268 & PREPARED \\
\hline 1269 & RELEASED \\
\hline 1270 & SENSITIVITY \\
\hline 1271 & BIRTH \\
\hline 1272 & STEPS \\
\hline 1273 & CONSCIOUSNESS \\
\hline 1274 & CORTICOSTEROIDS \\
\hline 1275 & DOCTOR \\
\hline 1276 & ENTER \\
\hline 1277 & MEAN \\
\hline 1278 & DIARRHEA \\
\hline 1279 & PHYSICIANS \\
\hline 1280 & ROUTE \\
\hline 1281 & THINGS \\
\hline 1282 & DISTURBANCES \\
\hline 1283 & HEPATIC \\
\hline 1284 & INVOLVEMENT \\
\hline
\end{tabular}




\begin{tabular}{|c|c|}
\hline 1285 & MODERATE \\
\hline 1286 & RECEPTORS \\
\hline 1287 & THROMBOSIS \\
\hline 1288 & CARBON \\
\hline 1289 & INCLUDED \\
\hline 1290 & LATE \\
\hline 1291 & PRACTITIONERS \\
\hline 1292 & SUFFICIENT \\
\hline 1293 & UTERUS \\
\hline 1294 & PROTECTIVE \\
\hline 1295 & REFERS \\
\hline 1296 & ENHANCE \\
\hline 1297 & MATTER \\
\hline 1298 & STOMA \\
\hline 1299 & TEACH \\
\hline 1300 & THINK \\
\hline 1301 & TYPICALLY \\
\hline 1302 & ACIDS \\
\hline 1303 & PRIVACY \\
\hline 1304 & SHIFT \\
\hline 1305 & SUBSEQUENT \\
\hline 1306 & IMPROVING \\
\hline 1307 & BODIES \\
\hline 1308 & FIELD \\
\hline 1309 & OBESITY \\
\hline 1310 & SCHOOL \\
\hline 1311 & WEAR \\
\hline 1312 & AID \\
\hline 1313 & ANALGESICS \\
\hline 1314 & RARE \\
\hline 1315 & REACH \\
\hline 1316 & TENDERNESS \\
\hline 1317 & INABILITY \\
\hline 1318 & OPENING \\
\hline 1319 & AGAIN \\
\hline 1320 & EFFORTS \\
\hline 1321 & TRANSFER \\
\hline 1322 & TRANSMITTED \\
\hline 1323 & GUIDE \\
\hline 1324 & REPRODUCTIVE \\
\hline 1325 & STIMULATE \\
\hline 1326 & ANAEMIA \\
\hline 1327 & RISE \\
\hline
\end{tabular}

\begin{tabular}{|c|c|}
\hline 1328 & SHOULDER \\
\hline 1329 & ACCOUNTABILITY \\
\hline 1330 & CLEARLY \\
\hline 1331 & COMPARED \\
\hline 1332 & CULTURES \\
\hline 1333 & HYPOTENSION \\
\hline 1334 & SYMPTOM \\
\hline 1335 & CONSTANT \\
\hline 1336 & CRANIAL \\
\hline 1337 & ABILITIES \\
\hline 1338 & ATTITUDES \\
\hline 1339 & DIAPHRAGM \\
\hline 1340 & FEEDBACK \\
\hline 1341 & HOLD \\
\hline 1342 & OBJECTIVES \\
\hline 1343 & SUGGEST \\
\hline 1344 & CAPILLARY \\
\hline 1345 & INFANTS \\
\hline 1346 & SCHEDULE \\
\hline 1347 & WALKING \\
\hline 1348 & ALMOST \\
\hline 1349 & ASTHMA \\
\hline 1350 & FACIAL \\
\hline 1351 & LARGER \\
\hline 1352 & SHAPE \\
\hline 1353 & SKELETAL \\
\hline 1354 & STIMULI \\
\hline 1355 & SYMPATHETIC \\
\hline 1356 & VESSEL \\
\hline 1357 & FALLS \\
\hline 1358 & OBSERVE \\
\hline 1359 & CONSIDERATIONS \\
\hline 1360 & CONTEXT \\
\hline 1361 & DESPITE \\
\hline 1362 & DOSAGE \\
\hline 1363 & OBJECTS \\
\hline 1364 & TENDS \\
\hline 1365 & TRADITIONAL \\
\hline 1366 & CRITERIA \\
\hline 1367 & DYING \\
\hline 1368 & DYSPNEA \\
\hline 1369 & FINGERS \\
\hline 1370 & POLITICAL \\
\hline
\end{tabular}

\begin{tabular}{|c|c|}
\hline 1371 & SELECTED \\
\hline 1372 & ACTS \\
\hline 1373 & BLOCK \\
\hline 1374 & EPISODES \\
\hline 1375 & INNER \\
\hline 1376 & SAMPLE \\
\hline 1377 & TOXIC \\
\hline 1378 & TRANSFUSION \\
\hline 1379 & ABSORBED \\
\hline 1380 & DEPEND \\
\hline 1381 & INVASIVE \\
\hline 1382 & OLD \\
\hline 1383 & CORTEX \\
\hline 1384 & MORPHINE \\
\hline 1385 & SUSPECTED \\
\hline 1386 & TONE \\
\hline 1387 & ADVICE \\
\hline 1388 & ATTACHED \\
\hline 1389 & CENTERS \\
\hline 1390 & COMBINED \\
\hline 1391 & INDEPENDENT \\
\hline 1392 & INTERVALS \\
\hline 1393 & INVOLVING \\
\hline 1394 & OCCASIONALLY \\
\hline 1395 & POSSIBLY \\
\hline 1396 & DELAYED \\
\hline 1397 & INITIATED \\
\hline 1398 & KNEE \\
\hline 1399 & YOUNGER \\
\hline 1400 & SITTING \\
\hline 1401 & CANCERS \\
\hline 1402 & MINUTE \\
\hline 1403 & SURROUNDING \\
\hline 1404 & WANT \\
\hline 1405 & CIRCULATORY \\
\hline 1406 & DESTRUCTION \\
\hline 1407 & EVALUATED \\
\hline 1408 & INTERFERE \\
\hline 1409 & OPTIONS \\
\hline 1410 & SUGGESTS \\
\hline 1411 & COUNSELING \\
\hline 1412 & NUTRIENTS \\
\hline 1413 & PANCREAS \\
\hline
\end{tabular}




\begin{tabular}{|c|c|}
\hline 1414 & SELFESTEEM \\
\hline 1415 & UNDERGOING \\
\hline 1416 & ARTHRITIS \\
\hline 1417 & BEHAVIOUR \\
\hline 1418 & CONTRACT \\
\hline 1419 & BENIGN \\
\hline 1420 & INTENSITY \\
\hline 1421 & LOCATED \\
\hline 1422 & MINIMAL \\
\hline 1423 & NEAR \\
\hline 1424 & PASS \\
\hline 1425 & SLIGHTLY \\
\hline 1426 & ABNORMALITIES \\
\hline 1427 & ASPECT \\
\hline 1428 & CONTAINING \\
\hline 1429 & FALL \\
\hline 1430 & INTEREST \\
\hline 1431 & SCALE \\
\hline 1432 & ADMINISTER \\
\hline 1433 & FOCUSES \\
\hline 1434 & INFARCTION \\
\hline 1435 & LOBE \\
\hline 1436 & ANALGESIA \\
\hline 1437 & DEFICITS \\
\hline 1438 & DIMINISHED \\
\hline 1439 & HELPING \\
\hline 1440 & COMFORTABLE \\
\hline 1441 & FOLLOWS \\
\hline 1442 & HOT \\
\hline 1443 & SOMEONE \\
\hline 1444 & TRY \\
\hline 1445 & CREATE \\
\hline 1446 & DEFICIT \\
\hline 1447 & ENTIRE \\
\hline 1448 & MEAL \\
\hline 1449 & PERCEIVED \\
\hline 1450 & TRACTION \\
\hline 1451 & CAST \\
\hline 1452 & EXPOSED \\
\hline 1453 & HELD \\
\hline 1454 & PREVENTIVE \\
\hline 1455 & VAGINA \\
\hline 1456 & BEHIND \\
\hline
\end{tabular}

\begin{tabular}{|c|c|}
\hline 1457 & MORTALITY \\
\hline 1458 & PROMPTLY \\
\hline 1459 & SERVE \\
\hline 1460 & SHOWS \\
\hline 1461 & SWALLOWING \\
\hline 1462 & TODAY \\
\hline 1463 & WORKERS \\
\hline 1464 & ANTIGEN \\
\hline 1465 & DESIGNED \\
\hline 1466 & DIAGNOSED \\
\hline 1467 & EASE \\
\hline 1468 & INDEPENDENCE \\
\hline 1469 & PLACEMENT \\
\hline 1470 & CONTENTS \\
\hline 1471 & NODE \\
\hline 1472 & OPIOID \\
\hline 1473 & PRINCIPLE \\
\hline 1474 & SALINE \\
\hline 1475 & TOWARDS \\
\hline 1476 & EARLIER \\
\hline 1477 & FEARS \\
\hline 1478 & LIMITS \\
\hline 1479 & MAINTAINS \\
\hline 1480 & PERMISSION \\
\hline 1481 & REGULARLY \\
\hline 1482 & EMPHASIS \\
\hline 1483 & EXPECTATIONS \\
\hline 1484 & FRIENDS \\
\hline 1485 & LOOK \\
\hline 1486 & MANNER \\
\hline 1487 & MUCUS \\
\hline 1488 & PARENTERAL \\
\hline 1489 & STEM \\
\hline 1490 & ANOREXIA \\
\hline 1491 & ASPIRIN \\
\hline 1492 & COLLECTION \\
\hline 1493 & EVALUATING \\
\hline 1494 & OSTEOPOROSIS \\
\hline 1495 & SPHINCTER \\
\hline 1496 & VERBAL \\
\hline 1497 & WEAK \\
\hline 1498 & EAT \\
\hline 1499 & HALF \\
\hline
\end{tabular}

\begin{tabular}{|c|c|}
\hline 1500 & LIFETHREATENING \\
\hline 1501 & MAINLY \\
\hline 1502 & PERSISTENT \\
\hline 1503 & TEMPORARY \\
\hline 1504 & ALTER \\
\hline 1505 & AORTA \\
\hline 1506 & COMPONENT \\
\hline 1507 & DESCRIBES \\
\hline 1508 & EVER \\
\hline 1509 & BEYOND \\
\hline 1510 & CONDUCT \\
\hline 1511 & GRIEF \\
\hline 1512 & POINTS \\
\hline 1513 & TRANSPLANTATION \\
\hline 1514 & URETHRA \\
\hline 1515 & VISITS \\
\hline 1516 & ANTI \\
\hline 1517 & CONCEPT \\
\hline 1518 & OUTPATIENT \\
\hline 1519 & STANDING \\
\hline 1520 & SUPPORTIVE \\
\hline 1521 & TOPICAL \\
\hline 1522 & CONSIDERABLE \\
\hline 1523 & FAR \\
\hline 1524 & CATHETERS \\
\hline 1525 & DECREASING \\
\hline 1526 & DEMONSTRATES \\
\hline 1527 & EXAMINED \\
\hline 1528 & FIBROUS \\
\hline 1529 & FINANCIAL \\
\hline 1530 & GOVERNMENT \\
\hline 1531 & POTENTIALLY \\
\hline 1532 & SUICIDE \\
\hline 1533 & BECOMING \\
\hline 1534 & DIURETICS \\
\hline 1535 & MICROORGANISMS \\
\hline 1536 & PERITONEAL \\
\hline 1537 & PLATELETS \\
\hline 1538 & RESIDUAL \\
\hline 1539 & RESPIRATIONS \\
\hline 1540 & STOP \\
\hline 1541 & ACCEPT \\
\hline 1542 & DONOR \\
\hline
\end{tabular}




\begin{tabular}{|c|c|}
\hline 1543 & INTERACTIONS \\
\hline 1544 & LEAVE \\
\hline 1545 & MARKED \\
\hline 1546 & PRIMARILY \\
\hline 1547 & READILY \\
\hline 1548 & ABSCESS \\
\hline 1549 & AUTONOMY \\
\hline 1550 & EXPRESS \\
\hline 1551 & GENE \\
\hline 1552 & LINES \\
\hline 1553 & PERFORMING \\
\hline 1554 & PUMP \\
\hline 1555 & SUBCUTANEOUS \\
\hline 1556 & VOLUNTARY \\
\hline 1557 & WASTE \\
\hline 1558 & AVERAGE \\
\hline 1559 & CAREGIVER \\
\hline 1560 & EDUCATIONAL \\
\hline 1561 & EXCRETION \\
\hline 1562 & FLEXION \\
\hline 1563 & INCREASINGLY \\
\hline 1564 & MEDIA \\
\hline 1565 & PASSES \\
\hline 1566 & RELATE \\
\hline 1567 & THIN \\
\hline 1568 & TREATING \\
\hline 1569 & ACCOMPANIED \\
\hline 1570 & ALREADY \\
\hline 1571 & BODYS \\
\hline 1572 & IDENTIFICATION \\
\hline 1573 & OCCUPATIONAL \\
\hline 1574 & OPIOIDS \\
\hline 1575 & CLOTHING \\
\hline 1576 & POSITIONS \\
\hline 1577 & SOMETHING \\
\hline 1578 & SPUTUM \\
\hline 1579 & SUPPLIES \\
\hline 1580 & TALK \\
\hline 1581 & ANAL \\
\hline 1582 & AVOIDING \\
\hline 1583 & COUNTRIES \\
\hline 1584 & MEMORY \\
\hline 1585 & NECROSIS \\
\hline
\end{tabular}

\begin{tabular}{|c|c|}
\hline 1586 & OPTIMAL \\
\hline 1587 & REFLEXES \\
\hline 1588 & ARMS \\
\hline 1589 & CLASSIFIED \\
\hline 1590 & RECORDS \\
\hline 1591 & STAY \\
\hline 1592 & SUPERFICIAL \\
\hline 1593 & ACQUIRED \\
\hline 1594 & COMMUNICATE \\
\hline 1595 & GIVES \\
\hline 1596 & LEADER \\
\hline 1597 & WIDELY \\
\hline 1598 & DIGESTIVE \\
\hline 1599 & HOSPICE \\
\hline 1600 & ANGER \\
\hline 1601 & ASSUME \\
\hline 1602 & CHOICES \\
\hline 1603 & KIND \\
\hline 1604 & PROMOTES \\
\hline 1605 & TACHYCARDIA \\
\hline 1606 & AFFECTING \\
\hline 1607 & FINGER \\
\hline 1608 & GRAVITY \\
\hline 1609 & PROGNOSIS \\
\hline 1610 & RECTAL \\
\hline 1611 & UNKNOWN \\
\hline 1612 & CARCINOMA \\
\hline 1613 & CAREGIVERS \\
\hline 1614 & INSTEAD \\
\hline 1615 & VISIT \\
\hline 1616 & ALTERATIONS \\
\hline 1617 & DEVELOPMENTAL \\
\hline 1618 & ENZYME \\
\hline 1619 & HYPOXIA \\
\hline 1620 & LENS \\
\hline 1621 & MORNING \\
\hline 1622 & PARTNER \\
\hline 1623 & SUCCESS \\
\hline 1624 & COVERED \\
\hline 1625 & INTRODUCED \\
\hline 1626 & RECEIVED \\
\hline 1627 & UNIQUE \\
\hline 1628 & BELIEVE \\
\hline
\end{tabular}

\begin{tabular}{|c|c|}
\hline 1629 & DIOXIDE \\
\hline 1630 & FRONT \\
\hline 1631 & MINOR \\
\hline 1632 & MUSCULAR \\
\hline 1633 & PROVISION \\
\hline 1634 & ORIGIN \\
\hline 1635 & PRIVATE \\
\hline 1636 & STIMULATES \\
\hline 1637 & COMPLICATION \\
\hline 1638 & DAMAGED \\
\hline 1639 & ENZYMES \\
\hline 1640 & PANCREATIC \\
\hline 1641 & READING \\
\hline 1642 & CHAIR \\
\hline 1643 & EXTENDED \\
\hline 1644 & GENDER \\
\hline 1645 & LAYERS \\
\hline 1646 & LIES \\
\hline 1647 & REFERRAL \\
\hline 1648 & SIMPLY \\
\hline 1649 & VOICE \\
\hline 1650 & ASSESSMENTS \\
\hline 1651 & BATH \\
\hline 1652 & GENETICS \\
\hline 1653 & SINUS \\
\hline 1654 & STABLE \\
\hline 1655 & SKULL \\
\hline 1656 & SUBJECT \\
\hline 1657 & VIEWED \\
\hline 1658 & WITHDRAWAL \\
\hline 1659 & COMES \\
\hline 1660 & DIFFER \\
\hline 1661 & GRAFT \\
\hline 1662 & INSUFFICIENCY \\
\hline 1663 & ORGANIZATIONAL \\
\hline 1664 & PROFESSION \\
\hline 1665 & ANESTHETIC \\
\hline 1666 & ATTACK \\
\hline 1667 & CAPILLARIES \\
\hline 1668 & INSTRUCTION \\
\hline 1669 & MODELS \\
\hline 1670 & PROGRESSION \\
\hline 1671 & REGION \\
\hline
\end{tabular}




\begin{tabular}{|c|c|}
\hline 1672 & ADMINISTERING \\
\hline 1673 & COMPHCATIONS \\
\hline 1674 & DEFINITION \\
\hline 1675 & DEMAND \\
\hline 1676 & DIRECTION \\
\hline 1677 & MAXIMUM \\
\hline 1678 & NOTES \\
\hline 1679 & PATHOPHYSIOLOGY \\
\hline 1680 & PLANNED \\
\hline 1681 & REDNESS \\
\hline 1682 & VOIDING \\
\hline 1683 & ACCEPTABLE \\
\hline 1684 & AORTIC \\
\hline 1685 & FORWARD \\
\hline 1686 & HEPARIN \\
\hline 1687 & PREPARE \\
\hline 1688 & SEDATION \\
\hline 1689 & SEPSIS \\
\hline 1690 & CLINIC \\
\hline 1691 & DIABETIC \\
\hline 1692 & HEARD \\
\hline 1693 & KEEPING \\
\hline 1694 & MANAGED \\
\hline 1695 & OCCURRED \\
\hline 1696 & REPAIR \\
\hline 1697 & ACIDOSIS \\
\hline 1698 & ALLOWING \\
\hline 1699 & COMMUNITYBASED \\
\hline 1700 & EXTREMELY \\
\hline 1701 & START \\
\hline 1702 & STEP \\
\hline 1703 & COMPUTER \\
\hline 1704 & INTRACRANIAL \\
\hline 1705 & READ \\
\hline 1706 & SUPERIOR \\
\hline 1707 & DRAIN \\
\hline 1708 & HABITS \\
\hline 1709 & RECTUM \\
\hline 1710 & RECURRENT \\
\hline 1711 & REGISTERED \\
\hline 1712 & THOUGH \\
\hline 1713 & CHLORIDE \\
\hline 1714 & ECONOMIC \\
\hline
\end{tabular}

\begin{tabular}{|c|c|}
\hline 1715 & EXCRETED \\
\hline 1716 & INSTANCES \\
\hline 1717 & LUMBAR \\
\hline 1718 & MONITORS \\
\hline 1719 & PARTICIPATION \\
\hline 1720 & VALVES \\
\hline 1721 & APPLYING \\
\hline 1722 & DOCUMENT \\
\hline 1723 & EXPECT \\
\hline 1724 & FORCES \\
\hline 1725 & IMMUNITY \\
\hline 1726 & LYING \\
\hline 1727 & PERHAPS \\
\hline 1728 & EXIST \\
\hline 1729 & EXPLORE \\
\hline 1730 & HEMOGLOBIN \\
\hline 1731 & INHIBITORS \\
\hline 1732 & SMALLER \\
\hline 1733 & SUGGESTED \\
\hline 1734 & ANTIBODY \\
\hline 1735 & DENTAL \\
\hline 1736 & EXCEPT \\
\hline 1737 & INTACT \\
\hline 1738 & INTENSIVE \\
\hline 1739 & POSSIBILITY \\
\hline 1740 & PRODUCING \\
\hline 1741 & BEGINNING \\
\hline 1742 & HARD \\
\hline 1743 & MOLECULES \\
\hline 1744 & OPPORTUNITIES \\
\hline 1745 & PARALYSIS \\
\hline 1746 & REQUIRING \\
\hline 1747 & SHARE \\
\hline 1748 & APPETITE \\
\hline 1749 & ATROPHY \\
\hline 1750 & CARTILAGE \\
\hline 1751 & COMPETENT \\
\hline 1752 & FIBERS \\
\hline 1753 & HAEMORRHAGE \\
\hline 1754 & MOIST \\
\hline 1755 & PHARMACOLOGIC \\
\hline 1756 & REFLECT \\
\hline 1757 & SPLEEN \\
\hline
\end{tabular}

\begin{tabular}{|c|c|}
\hline 1758 & TRACHEA \\
\hline 1759 & AGES \\
\hline 1760 & CLOTTING \\
\hline 1761 & CURRENTLY \\
\hline 1762 & EXPERIENCING \\
\hline 1763 & FEATURES \\
\hline 1764 & IMMOBILITY \\
\hline 1765 & IMPLEMENTATION \\
\hline 1766 & ISSUE \\
\hline 1767 & MILK \\
\hline 1768 & OBJECTIVE \\
\hline 1769 & TUBING \\
\hline 1770 & BASELINE \\
\hline 1771 & CHOOSE \\
\hline 1772 & DIFFICULTIES \\
\hline 1773 & INTERMITTENT \\
\hline 1774 & OBJECT \\
\hline 1775 & OUTER \\
\hline 1776 & SURFACES \\
\hline 1777 & CODE \\
\hline 1778 & CONDUCTED \\
\hline 1779 & MELLITUS \\
\hline 1780 & MET \\
\hline 1781 & TOP \\
\hline 1782 & CLASSIFICATION \\
\hline 1783 & DETECTED \\
\hline 1784 & DOCTORS \\
\hline 1785 & MILLION \\
\hline 1786 & POSITIONING \\
\hline 1787 & PROBABLY \\
\hline 1788 & RECEPTOR \\
\hline 1789 & TARGET \\
\hline 1790 & WARD \\
\hline 1791 & ATTEMPTS \\
\hline 1792 & COMPOSED \\
\hline 1793 & COVER \\
\hline 1794 & SAY \\
\hline 1795 & UTERINE \\
\hline 1796 & ABSENT \\
\hline 1797 & BUDGET \\
\hline 1798 & CALL \\
\hline 1799 & ENDS \\
\hline 1800 & ILLNESSES \\
\hline
\end{tabular}




\begin{tabular}{|c|c|}
\hline 1801 & LARGELY \\
\hline 1802 & NUMBERS \\
\hline 1803 & PROMPT \\
\hline 1804 & TERMINAL \\
\hline 1805 & UNIVERSAL \\
\hline 1806 & ADMITTED \\
\hline 1807 & DEFICIENT \\
\hline 1808 & EXTREME \\
\hline 1809 & INJURED \\
\hline 1810 & PORTAL \\
\hline 1811 & ADMISSION \\
\hline 1812 & BARRIER \\
\hline 1813 & CONTAINER \\
\hline 1814 & REAL \\
\hline 1815 & RECOGNITION \\
\hline 1816 & THROAT \\
\hline 1817 & DESIRE \\
\hline 1818 & PELVIS \\
\hline 1819 & PHASES \\
\hline 1820 & RELATIVE \\
\hline 1821 & CHOLESTEROL \\
\hline 1822 & CIRCULATING \\
\hline 1823 & DELEGATION \\
\hline 1824 & ENCOURAGES \\
\hline 1825 & IDENTITY \\
\hline 1826 & TECHNOLOGY \\
\hline 1827 & ADHERENCE \\
\hline 1828 & BUMS \\
\hline 1829 & ELASTIC \\
\hline 1830 & INTRODUCTION \\
\hline 1831 & MEASUREMENT \\
\hline 1832 & PRIORITIES \\
\hline 1833 & ALLOWED \\
\hline 1834 & ANGINA \\
\hline 1835 & ANTIGENS \\
\hline 1836 & IDENTIFIES \\
\hline 1837 & IRREGULAR \\
\hline 1838 & OFFERED \\
\hline 1839 & ORDERS \\
\hline 1840 & RUPTURE \\
\hline 1841 & ADDED \\
\hline 1842 & BAG \\
\hline 1843 & BREAK \\
\hline
\end{tabular}

\begin{tabular}{|c|c|}
\hline 1844 & CHALLENGE \\
\hline 1845 & CHARGE \\
\hline 1846 & DUCTS \\
\hline 1847 & FRAMEWORK \\
\hline 1848 & OPERATIVE \\
\hline 1849 & PRESSURES \\
\hline 1850 & SAID \\
\hline 1851 & SPECIALIST \\
\hline 1852 & ACTUALLY \\
\hline 1853 & ALLY \\
\hline 1854 & COMA \\
\hline 1855 & CONSIST \\
\hline 1856 & TASTE \\
\hline 1857 & TRUST \\
\hline 1858 & AUDITORY \\
\hline 1859 & FAIL \\
\hline 1860 & LIMIT \\
\hline 1861 & MIND \\
\hline 1862 & PARTNERS \\
\hline 1863 & REGARDLESS \\
\hline 1864 & SIGNIFICANTLY \\
\hline 1865 & STUDENT \\
\hline 1866 & COLLABORATIVE \\
\hline 1867 & DYSRHYTHMIAS \\
\hline 1868 & KINDS \\
\hline 1869 & MENSTRUAL \\
\hline 1870 & SECONDS \\
\hline 1871 & STONES \\
\hline 1872 & ACCOUNTABLE \\
\hline 1873 & ASSISTED \\
\hline 1874 & CHARACTERISTIC \\
\hline 1875 & COMPLETED \\
\hline 1876 & DEPTH \\
\hline 1877 & DETAILED \\
\hline 1878 & INTERESTS \\
\hline 1879 & LIMITATIONS \\
\hline 1880 & SCAN \\
\hline 1881 & SCOPE \\
\hline 1882 & THERAPIST \\
\hline 1883 & ASSISTIVE \\
\hline 1884 & CERVIX \\
\hline 1885 & CONSISTENT \\
\hline 1886 & CUFF \\
\hline
\end{tabular}

\begin{tabular}{|c|c|}
\hline 1887 & ESOPHAGEAL \\
\hline 1888 & LYMPHOCYTES \\
\hline 1889 & MEETING \\
\hline 1890 & STARTED \\
\hline 1891 & SYNTHESIS \\
\hline 1892 & AUTONOMIC \\
\hline 1893 & GREATLY \\
\hline 1894 & HEAVY \\
\hline 1895 & HYDRATION \\
\hline 1896 & MUSCULOSKELETAL \\
\hline 1897 & PROGRESSES \\
\hline 1898 & SHARED \\
\hline 1899 & CREASED \\
\hline 1900 & CURE \\
\hline 1901 & INFANT \\
\hline 1902 & INFERIOR \\
\hline 1903 & LISTED \\
\hline 1904 & SPINE \\
\hline 1905 & DEMENTIA \\
\hline 1906 & DEPENDENCE \\
\hline 1907 & FAMILIAR \\
\hline 1908 & ORIENTATION \\
\hline 1909 & PORT \\
\hline 1910 & SUSCEPTIBLE \\
\hline 1911 & AMINO \\
\hline 1912 & ESTIMATED \\
\hline 1913 & IMAGING \\
\hline 1914 & INFLUENCED \\
\hline 1915 & OESOPHAGUS \\
\hline 1916 & PRIORITY \\
\hline 1917 & DEGREES \\
\hline 1918 & INITIATE \\
\hline 1919 & MAJORITY \\
\hline 1920 & NASOGASTRIC \\
\hline 1921 & OCCURRING \\
\hline 1922 & WAVES \\
\hline 1923 & ASSISTING \\
\hline 1924 & BONY \\
\hline 1925 & DELIVERED \\
\hline 1926 & EXPRESSION \\
\hline 1927 & RECURRENCE \\
\hline 1928 & CONGENITAL \\
\hline 1929 & ELECTROLYTES \\
\hline
\end{tabular}




\begin{tabular}{|c|c|}
\hline 1930 & FEEDINGS \\
\hline 1931 & FLAT \\
\hline 1932 & LOCALIZED \\
\hline 1933 & VIRUSES \\
\hline 1934 & FIT \\
\hline 1935 & GROWING \\
\hline 1936 & HEIGHT \\
\hline 1937 & INSTITUTIONS \\
\hline 1938 & ORGANISM \\
\hline 1939 & OVARIAN \\
\hline 1940 & PROXIMAL \\
\hline 1941 & WELLNESS \\
\hline 1942 & ACCUMULATION \\
\hline 1943 & ASSISTS \\
\hline 1944 & DOCUMENTATION \\
\hline 1945 & EQUAL \\
\hline 1946 & GENTLY \\
\hline 1947 & IDEAS \\
\hline 1948 & INTERCOURSE \\
\hline 1949 & OBTAINING \\
\hline 1950 & PALPATION \\
\hline 1951 & RECEIVES \\
\hline 1952 & TOBACCO \\
\hline 1953 & ADVANCE \\
\hline 1954 & ANXIOUS \\
\hline 1955 & BURN \\
\hline 1956 & CHEMICALS \\
\hline 1957 & CONDUCTION \\
\hline 1958 & FLOOR \\
\hline 1959 & MASK \\
\hline 1960 & ORALLY \\
\hline 1961 & RATIONAL \\
\hline 1962 & RESPIRATION \\
\hline 1963 & RULE \\
\hline 1964 & SEPARATE \\
\hline 1965 & SKILLED \\
\hline 1966 & TRUE \\
\hline 1967 & ADOLESCENTS \\
\hline 1968 & DISTURBANCE \\
\hline 1969 & FORMAL \\
\hline 1970 & HYPOTHALAMUS \\
\hline 1971 & REMAINING \\
\hline 1972 & SEIZURE \\
\hline
\end{tabular}

\begin{tabular}{|c|c|}
\hline 1973 & SEXUALLY \\
\hline 1974 & APPRAISAL \\
\hline 1975 & DEALING \\
\hline 1976 & DUODENUM \\
\hline 1977 & FELT \\
\hline 1978 & FUNDAMENTAL \\
\hline 1979 & GREATEST \\
\hline 1980 & IMPLEMENTING \\
\hline 1981 & ISOLATION \\
\hline 1982 & TRANS \\
\hline 1983 & BARRIERS \\
\hline 1984 & CHAMBER \\
\hline 1985 & EXAMINE \\
\hline 1986 & FEELS \\
\hline 1987 & GUIDANCE \\
\hline 1988 & INDICATORS \\
\hline 1989 & INSIDE \\
\hline 1990 & MOTHER \\
\hline 1991 & RESTORE \\
\hline 1992 & TURNING \\
\hline 1993 & DUTIES \\
\hline 1994 & EASY \\
\hline 1995 & ENLARGED \\
\hline 1996 & IMPLEMENT \\
\hline 1997 & INSURANCE \\
\hline 1998 & PRESENTED \\
\hline 1999 & PUNCTURE \\
\hline 2000 & TENDENCY \\
\hline 2001 & BRANCHES \\
\hline 2002 & BRING \\
\hline 2003 & CONSCIOUS \\
\hline 2004 & DISTRIBUTION \\
\hline 2005 & ENCOURAGING \\
\hline 2006 & ISCHEMIA \\
\hline 2007 & MOTIVATION \\
\hline 2008 & OPTIC \\
\hline 2009 & PARTICIPANTS \\
\hline 2010 & PROGRAMME \\
\hline 2011 & VERTEBRAL \\
\hline 2012 & WAVE \\
\hline 2013 & ANSWER \\
\hline 2014 & BATHING \\
\hline 2015 & DIARRHOEA \\
\hline
\end{tabular}

\begin{tabular}{|c|c|}
\hline 2016 & DRINK \\
\hline 2017 & FIRM \\
\hline 2018 & IMBALANCE \\
\hline 2019 & JAUNDICE \\
\hline 2020 & LIQUID \\
\hline 2021 & NEURONES \\
\hline 2022 & NUMEROUS \\
\hline 2023 & PHARYNX \\
\hline 2024 & SERIES \\
\hline 2025 & STIMULATED \\
\hline 2026 & THREAT \\
\hline 2027 & TOXICITY \\
\hline 2028 & FOCUSED \\
\hline 2029 & MOVED \\
\hline 2030 & OBSERVATION \\
\hline 2031 & PENIS \\
\hline 2032 & POSTURE \\
\hline 2033 & PRESCRIPTION \\
\hline 2034 & REFER \\
\hline 2035 & SCIENTIFIC \\
\hline 2036 & SUB \\
\hline 2037 & TEAMS \\
\hline 2038 & TOES \\
\hline 2039 & DIFFERENCE \\
\hline 2040 & HIGHEST \\
\hline 2041 & INTERACTION \\
\hline 2042 & LOSSES \\
\hline 2043 & RESTRICTED \\
\hline 2044 & SHEET \\
\hline 2045 & STORED \\
\hline 2046 & VULNERABLE \\
\hline 2047 & CONTAMINATION \\
\hline 2048 & CREATED \\
\hline 2049 & DEFINE \\
\hline 2050 & DEPRESSED \\
\hline 2051 & FATTY \\
\hline 2052 & PERFORATION \\
\hline 2053 & RETINA \\
\hline 2054 & SECRETED \\
\hline 2055 & ACCEPTANCE \\
\hline 2056 & BILIARY \\
\hline 2057 & COLOUR \\
\hline 2058 & COMPETENCE \\
\hline
\end{tabular}




\begin{tabular}{|c|c|}
\hline 2059 & LUMEN \\
\hline 2060 & PLASTIC \\
\hline 2061 & PRACTITIONER \\
\hline 2062 & PSYCHIATRIC \\
\hline 2063 & STAND \\
\hline 2064 & TESTED \\
\hline 2065 & CONTRAINDICATED \\
\hline 2066 & DETAIL \\
\hline 2067 & INJECTED \\
\hline 2068 & INSPECT \\
\hline 2069 & ITEMS \\
\hline 2070 & LYMPHATIC \\
\hline 2071 & OPERATING \\
\hline 2072 & PULSES \\
\hline 2073 & RECENTLY \\
\hline 2074 & SEXUALITY \\
\hline 2075 & TYPICAL \\
\hline 2076 & ABORTION \\
\hline 2077 & ADJACENT \\
\hline 2078 & ADJUST \\
\hline 2079 & BELIEF \\
\hline 2080 & BELIEVED \\
\hline 2081 & EMPTY \\
\hline 2082 & EXPANSION \\
\hline 2083 & MIXED \\
\hline 2084 & PREVENTED \\
\hline 2085 & REALITY \\
\hline 2086 & SHORTNESS \\
\hline 2087 & SPEAK \\
\hline 2088 & SUPPLEMENTS \\
\hline 2089 & ACUITY \\
\hline 2090 & AMBULATORY \\
\hline 2091 & COLLECTED \\
\hline 2092 & INTERNATIONAL \\
\hline 2093 & LINING \\
\hline 2094 & PASSED \\
\hline 2095 & PURPOSES \\
\hline 2096 & REPLACED \\
\hline 2097 & STRATEGIC \\
\hline 2098 & TELEPHONE \\
\hline 2099 & ULCERATION \\
\hline 2100 & WORN \\
\hline 2101 & WRONG \\
\hline
\end{tabular}

\begin{tabular}{|c|c|}
\hline 2102 & CIRRHOSIS \\
\hline 2103 & DETECTION \\
\hline 2104 & DISCHARGED \\
\hline 2105 & DISTENTION \\
\hline 2106 & INDEX \\
\hline 2107 & PASSIVE \\
\hline 2108 & PROPERLY \\
\hline 2109 & PROPORTION \\
\hline 2110 & RELATIVES \\
\hline 2111 & SATISFACTION \\
\hline 2112 & ACTIVELY \\
\hline 2113 & BEDSIDE \\
\hline 2114 & FEWER \\
\hline 2115 & GENERALIZED \\
\hline 2116 & HOST \\
\hline 2117 & IMPULSE \\
\hline 2118 & SELFCONCEPT \\
\hline 2119 & URETHRAL \\
\hline 2120 & CHALLENGES \\
\hline 2121 & EDGE \\
\hline 2122 & ENABLE \\
\hline 2123 & EXTENDS \\
\hline 2124 & IODINE \\
\hline 2125 & LED \\
\hline 2126 & REPLACE \\
\hline 2127 & SAFELY \\
\hline 2128 & SLIGHT \\
\hline 2129 & SPECIMENS \\
\hline 2130 & VIEWS \\
\hline 2131 & ATTACKS \\
\hline 2132 & BIOLOGIC \\
\hline 2133 & CATEGORIES \\
\hline 2134 & CREATININE \\
\hline 2135 & DIVIDED \\
\hline 2136 & ETHNIC \\
\hline 2137 & EXAM \\
\hline 2138 & FORMING \\
\hline 2139 & MICROBES \\
\hline 2140 & REGIMENS \\
\hline 2141 & RELIEVING \\
\hline 2142 & TOLERATED \\
\hline 2143 & VENTRICLES \\
\hline 2144 & BURNING \\
\hline
\end{tabular}

2145 CONCENTRATIONS

2146 CONTAMINATED

2147 DELAY

2148 ESOPHAGUS

2149 FINALLY

2150 GETTING

2151 MEASUREMENTS

2152 NEUROLOGICAL

2153 OBVIOUS

2154 PREPARATIONS

2155 RECOGNIZED

2156 SUPPORTS

2157 VISIBLE 